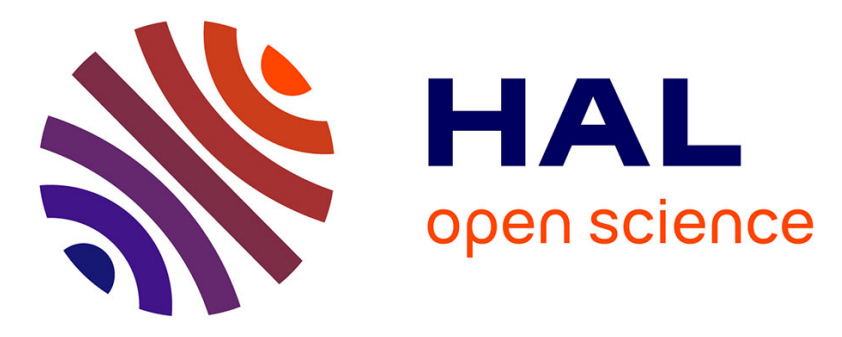

\title{
Theoretical and revisited experimentally retrieved He-broadened line parameters of carbon monoxide in the fundamental band
}

\author{
A. Predoi-Cross, K. Esteki, H. Rozario, H. Naseri, S. Latif, Franck Thibault, \\ V. Malathy Devi, M.A.H. Smith, A.W. Mantz
}

\section{To cite this version:}

A. Predoi-Cross, K. Esteki, H. Rozario, H. Naseri, S. Latif, et al.. Theoretical and revisited experimentally retrieved He-broadened line parameters of carbon monoxide in the fundamental band. Journal of Quantitative Spectroscopy and Radiative Transfer, 2016, 184, pp.322-340. 10.1016/j.jqsrt.2016.08.007 . hal-01370533

\section{HAL Id: hal-01370533 \\ https://hal.science/hal-01370533}

Submitted on 22 Sep 2016

HAL is a multi-disciplinary open access archive for the deposit and dissemination of scientific research documents, whether they are published or not. The documents may come from teaching and research institutions in France or abroad, or from public or private research centers.
L'archive ouverte pluridisciplinaire HAL, est destinée au dépôt et à la diffusion de documents scientifiques de niveau recherche, publiés ou non, émanant des établissements d'enseignement et de recherche français ou étrangers, des laboratoires publics ou privés. 
Authors' version. This paper has been published in JQSRT:

http://dx.doi.org/10.1016/j.jqsrt.2016.08.007

\title{
Theoretical and Revisited Experimentally Retrieved He-Broadened Line Parameters of Carbon Monoxide in the Fundamental Band
}

\author{
A. Predoi-Cross ${ }^{a}$, K. Esteki ${ }^{a}$, H. Rozario ${ }^{a}$, H. Naseria,b ${ }^{a, b}$ S. Latif ${ }^{\text {a,c }}$, F. Thibault ${ }^{d}$, V. Malathy Devi ${ }^{\mathrm{e}}$, \\ M.A.H. Smith , A.W. Mantz \\ ${ }^{a}$ Department of Physics and Astronomy, University of Lethbridge, Lethbridge, AB, T1K 6R4 Canada; \\ ${ }^{b}$ Present Address: Farmers Edge, Lethbridge, AB, Canada \\ ${ }^{c}$ Present Address: Department of Computer Science, University of Lethbridge, Lethbridge, AB, T1K \\ 6R4 Canada; \\ ${ }^{d}$ Institut de Physique de Rennes, UMR CNRS 6251, Université de Rennes 1, 35042 Rennes, France ; \\ ${ }^{e}$ Department of Physics, The College of William and Mary, Williamsburg, VA 23187, USA; \\ ${ }^{f}$ Science Directorate, NASA Langley Research Center, Hampton, VA 23681, USA; \\ ${ }^{g}$ Dept. of Physics, Astronomy and Geophysics, Connecticut College, New London, CT 06320, USA
}

Corresponding author:

Dr. Adriana Predoi-Cross

Department of Physics and Astronomy, University of Lethbridge, Lethbridge, AB, T1K 3M4 Canada

E-mail: adriana.predoicross@ uleth.ca Tel: 403-329-2697 Fax: 403-329-2057

Number of pages: 20 Number of Tables: 8 Number of Figures: 8

Short title: He-broadened CO line parameters in the $1 \leftarrow 0$ band: Revisited

Keywords: CO-He widths and shifts, Infrared spectra, Temperature dependences of widths and shifts, Line mixing, Spectral line shapes, speed dependence 


\begin{abstract}
We report revisited experimentally retrieved and theoretically calculated He-broadened Lorentz halfwidth coefficients and He- pressure-shift coefficients of 45 carbon monoxide transitions in the $1 \leftarrow 0$ band. The spectra analyzed in this study were recorded over a range of temperatures between 79 and 296 $\mathrm{K}$. The He-broadened line parameters and their temperature dependences were retrieved using a multispectrum nonlinear least squares analysis program. The line shape models used in this study include Voigt, speed dependent Voigt, Rautian (to take into account confinement narrowing) and Rautian with speed dependence, all with an asymmetric component added to account for weak line mixing effects. We were unable to retrieve the temperature dependence of line mixing coefficients. A classical method was used to determine the He-narrowing parameters while quantum dynamical calculations were performed to determine He-broadening and He-pressure shifts coefficients at different temperatures. The line mixing coefficients were also derived from the exponential power gap law and the energy corrected sudden approximation. The current measurements and theoretical results are compared with other published results, where appropriate.
\end{abstract}

\title{
I. INTRODUCTION
}

Carbon monoxide is present as a trace constituent in the Earth and planetary atmospheres, as well as in the interstellar medium (see for example, [1-4] and references therein). For decades, spectral line shape studies of self- and foreign-broadened ro-vibrational lines of $\mathrm{CO}$ have captivated the interest of experimentalists and theoreticians (e.g. [5-24]). Many of these spectroscopic studies have been triggered by the need for highly accurate line parameters for the interpretation of atmospheric spectra recorded by spectroscopic remote sensing instruments on ground, balloon or satellite platforms. A literature review of studies of the CO-He molecular system is given below.

One of the earlier line shape studies of carbon monoxide transitions was by Nerf and Sonnenberg [12] who investigated the carbon monoxide $\mathrm{R}(0)$ line located at $115 \mathrm{GHz}$. The authors measured the pressure broadening coefficients of $\mathrm{CO}$ broadened by hydrogen, deuterium, helium, neon, and argon for the $J=1 \leftarrow 0$ transition at three different temperatures ( $77 \mathrm{~K}, 195 \mathrm{~K}$ and $294 \mathrm{~K})$. Monnanteuil and Colmont [13] measured the foreign $\left(\mathrm{N}_{2}, \mathrm{O}_{2}\right.$, and $\left.\mathrm{He}\right)$ and self-broadening coefficients of carbon monoxide transitions in the millimeter range $(\sim 230 \mathrm{GHz})$ for temperatures in the 220 to $300 \mathrm{~K}$ range.

Boissoles et al. [14] have reported the theoretical line mixing coefficients of $\mathrm{CO}$ perturbed by $\mathrm{He}$ employing the infinite order sudden approximation (IOSA) and the energy corrected sudden approximation (ECSA) techniques. Green et al. [15] have computed the theoretical off-diagonal collision induced broadening cross sections for the $1 \leftarrow 0$ band of $\mathrm{CO}$ broadened by helium. The authors have compared experimental spectra of CO broadened by He recorded at temperatures of 78-292 K with spectra calculated line-by-line with contributions from theoretical line mixing. The helium and hydrogen broadened pressure - shift coefficients in carbon monoxide absorption spectra have been measured by Mannucci [16] for three ro-vibrational lines, $\mathrm{P}(8), \mathrm{R}(2)$, and $\mathrm{R}(14)$, of the fundamental band in the infrared region. An infrared heterodyne spectrometer with a spectral resolution of $20 \mathrm{MHz}$ was used in this study. 
The line mixing parameters and pressure-induced shift coefficients from room-temperature $\mathrm{CO}$ spectra broadened by $\mathrm{He}$ in the $1 \leftarrow 0$ and $2 \leftarrow 0$ bands have been measured and calculated by Thibault $e t$ al. [17]. Pressure broadening coefficients were also measured at room temperature and at $85 \mathrm{~K}$ in the $1 \leftarrow 0$ band in the latter study. The authors compared their experimental results with theoretical values [15] obtained from quantum dynamical calculations. Duggan et al. [18] have studied the high resolution $\left(10^{-4} \mathrm{~cm}^{-1}\right)$ infrared spectra of carbon monoxide diluted by $\mathrm{Xe}, \mathrm{N}_{2}$, and $\mathrm{He}$ in the intermediate pressure range. The high signal-to-noise ratio of the spectra $(\sim 2000: 1)$ allowed the authors to quantify the speed dependent collisional broadening effects on the retrieved line parameters. A Fourier transform spectrometer was used by Bouanich et al. [19] to record the absorption spectra of CO diluted by $\mathrm{He}, \mathrm{Ne}$, Ar, $\mathrm{Xe}, \mathrm{O}_{2}$, and $\mathrm{N}_{2}$ in the $1 \leftarrow 0$ band. The authors compared their experimental pressure-shift coefficients with the theoretical computations using a semi-classical model.

Henry et al. [20] used a tunable diode laser spectrometer system attached to a Michelson interferometer to measure the $\mathrm{R}(21), \mathrm{R}(23)$ and $\mathrm{R}(24)$ transitions of the fundamental band of $\mathrm{CO}$ perturbed by $\mathrm{He}, \mathrm{Ne}, \mathrm{Xe}$ and $\mathrm{N}_{2}$ at pressures between 10 and 600 Torr. The authors have retrieved the broadening, narrowing and pressure induced shift parameters using several line shape models such as the Voigt, Galatry (i.e. soft collision model) and Rautian (i.e. hard collision model). They also analyzed those spectra and compared the results with two new semi-empirical models generated by the convolution of Lorentzian with soft and hard collision models.

Beaky et al. [21] have measured the pressure broadening and shift parameters for the $\mathrm{R}(0)$ and $\mathrm{R}(1)$ pure rotational transitions of $\mathrm{CO}$ broadened by helium for temperatures between 1 and $600 \mathrm{~K}$ using the collisional cooling technique. Simultaneous close-coupling (CC) calculations of these parameters performed by Thachuk et al. [22] on different potential energy surfaces (PES) were shown to lead to good agreement with the measured values down to about $12 \mathrm{~K}$. Below an important mismatch between calculated and measured values is observed even using the best PES.

Sinclair et al. [23] published the CO-He broadening coefficients for 39 lines in the $\mathrm{P}$ and $\mathrm{R}$ branches of the fundamental band using a difference frequency laser spectrometer with spectral resolution of $2 \mathrm{MHz}$. This experimental setup allowed them to record spectra with signal-to-noise ratio of 3000:1 at 301.5 K using a temperature stabilized gas absorption cell. The authors investigated the absorption spectra using the soft collision model. The room temperature broadening and shift parameters of $\mathrm{CO}$ broadened by $\mathrm{He}$ and $\mathrm{Ar}$ were measured by Luo et al. [24] for several $\mathrm{R}$ - and P- branch transitions in the same band. The difference frequency laser spectrometer used in their study was a modified, 3-channel version of the spectrometer described in Ref. [23]. An overall good agreement was observed between the measured results and close coupling dynamical calculations performed on the best available interaction potential.

The McMath-Pierce Fourier transform spectrometer facility located at the National Solar Observatory on Kitt Peak was used by Mantz et al. [25] to record the spectra of CO perturbed by helium. In this study, the authors have investigated the Lorentz pressure-broadening and pressureinduced shift coefficients along with their temperature dependences of P and R branch lines in the 2010 to $2260 \mathrm{~cm}^{-1}$ region for a temperature range between 80 and $297 \mathrm{~K}$. The spectra were analyzed simultaneously using a multispectrum nonlinear least squares fitting technique [26]. 
Studies of helium broadened spectra of less-abundant isotopologues of carbon monoxide gas have also been carried out. For example, a Fourier transform spectrometer system composed of a tunable diode laser (TDL) coupled with a Michelson interferometer was employed by Mantz et al. [27] to measure the spectra of ${ }^{13} \mathrm{CO}$ broadened by helium over a wide range of temperatures from 11.5 to 298.6 $\mathrm{K}$. For the $\mathrm{P}(2)$ line of the fundamental, the authors determined the He-broadened half width coefficient and its temperature dependence. Henry et al. [28] have reported the ${ }^{13} \mathrm{CO}-\mathrm{He}$ broadening parameters taking confinement narrowing into account while fitting with the soft collision profile.

Thibault et al. [29] have reported the helium broadening coefficients of the $\mathrm{R}(0)$ and $\mathrm{P}(2)$ transitions of the fundamental band of ${ }^{13} \mathrm{CO}$ recorded at low temperatures (from $12 \mathrm{~K}$ up to room temperature) using a frequency stabilized diode laser system controlled by Michelson interferometer. The authors have determined the broadening coefficients by taking the narrowing effect into account using the Galatry profile. The fits using the soft collision model reproduced the experimental data better than the Voigt profile. The overall good agreement between measured pressure broadening and close coupling (CC) cross sections was observed to improve at temperatures below $30 \mathrm{~K}$.

In this paper, we present the results from a re-analysis of the He-broadened CO spectra in the fundamental band that were previously analyzed using the Voigt profile [25]. The line shape models used in the present multispectrum analysis include Voigt, Rautian, speed dependent Voigt, and Rautian with speed dependence. In all our analysis steps we have accounted for the weak line mixing effects using the Rosenkranz approach. We were unable to determine the temperature dependences of line mixing coefficients. Our present retrieved He-broadened parameters have been compared with published theoretical results and with previous measurements [17, 23-25].

\section{EXPERIMENTAL DETAILS}

The 19 spectra re-analyzed in this study were first studied in Ref. [25]. All spectra were recorded with a spectral resolution of $0.0052 \mathrm{~cm}^{-1}$ at the McMath-Pierce experimental facility on Kitt Peak Observatory, Arizona. Two temperature-controlled short absorption cells with path lengths of $0.011313 \pm 0.000002 \mathrm{~m}$ and $0.043050 \pm 0.0005 \mathrm{~m}$ and temperature stability of $\pm 0.01 \mathrm{~K}$ [25] were used to record spectra along with two InSb detectors, a cryogenic vacuum pump, and associated electronic instrumentation. The experimental conditions (temperature, pressure, volume mixing ratio, path length and the broadening gas) of the spectra are given in Table 2 of Ref. [25]. The readers are directed to Ref. [25] for a full set of experimental conditions and setup.

\section{SPECTROSCOPIC ANALYSIS}

For our re-analysis, the initial line positions and intensities for ${ }^{12} \mathrm{C}^{16} \mathrm{O}$ required for the multispectrum fits were taken from the HITRAN database [30,31], and the initial guesses for Hebroadened widths and shifts were taken from Ref. [25]. The careful calibration of the spectra described in Ref. [25] allowed us to obtain highly accurate line positions. The 19 spectra recorded at various temperatures and pressures, as in Ref. [25], were fitted simultaneously covering the entire spectral range 
of the fundamental band, using an interactive multispectrum fitting software [26], with no position and intensity constraints applied in the present study. To allow the readers to see the details of our spectra, in Fig. 1 we present the fit results for a section in the R-branch, nearby the R(9) line. The line parameters were retrieved using the Voigt, Rautian, speed dependent Voigt and Rautian with speed dependence line shape models. An asymmetric component that accounts for weak line mixing effects was added to the model profiles. The line mixing formalism will not be detailed here (see for example Refs. [17,32,33] and references therein).

We report retrievals for He-broadened line shape parameters and where possible, we provide their temperature dependences. The equations governing the retrievals of these line parameters are presented below [25]:

$$
\begin{gathered}
\gamma(p, T)=p \times\left[\gamma_{H e}^{0}\left(p_{0}, T_{0}\right) \times(1-\chi) \times\left[\frac{T_{0}}{T}\right]^{n_{1}}+\gamma_{\text {self }}^{0}\left(p_{0}, T_{0}\right) \times \chi \times\left[\frac{T_{0}}{T}\right]^{n_{2}}\right], \\
v(T)=v_{0}+p \times\left[\delta_{H e}^{0}(T) \times(1-\chi)+\delta_{\text {self }}^{0}(T) \times \chi\right], \\
\delta_{H e}^{0}(T)=\delta_{H e}^{0}\left(T_{0}\right)+\delta_{H e}^{\prime} \times\left(T-T_{0}\right) .
\end{gathered}
$$

In Eqs. (1) to (3), $\gamma^{0}$ and $\delta^{0}$ are the broadening and shift coefficients at the reference temperature $T_{0}$ (296 $\mathrm{K})$ and pressure $p_{o}(1 \mathrm{~atm}) ; \gamma(p, T)$ represents the Lorentz Half Width measured at Half Maximum (HWHM) at temperature $T$ and pressure $p ; \chi$ is the volume mixing ratio of CO; $n_{1}$ and $n_{2}$ are the temperature dependence exponents of (helium and self) broadening coefficients, respectively; $\delta^{\prime}$ corresponds to the temperature dependence of the pressure-induced shift coefficient [25].

An effect that appears under certain experimental conditions is the collisional narrowing of the line shape. For all molecules, in the pressure regime where the average path of the free moving molecules becomes equal to or less than the incident light's wavelength, the spectral lines have been observed to be narrower than predicted by the Voigt line shape. The pressure range for which this effect is observed is known as the "narrowing regime" and the effect is also called the Dicke narrowing effect. The narrowing effect has been included in the present multispectrum fit using the line shape described in Refs. [22-24] of Ref. [32]:

$$
\kappa(v)=\operatorname{Re}\left(\frac{E(x, y+e)}{1-\sqrt{\pi e E(x, y+e)}}\right),
$$

where, the parameter $e$ is the collisional narrowing parameter, $E(x, y)=K(x, y)+i L(x, y)$ is the complex error function, and Re means the real part. Benner et al. [26] present how the fits using the Voigt profile are handled in the multispectrum analysis.

The line broadening and pressure-induced shifts are known to be affected by the velocity of optically active gas molecules. It is possible to take into account the asymmetry due to the MaxwellBoltzmann velocity distribution in the line shape while modeling the spectra employing the speed dependent Voigt profile. The speed dependent line parameters can be determined from simultaneous fitting of several spectra using the nonlinear least square multispectrum fitting program with either the speed dependent Voigt line shape, or the Rautian line shape model including speed dependence. The 
speed dependence of the pressure broadening coefficients is accounted using a quadratic dependence on absolute velocity $v$ of the optically active molecule [34-38]:

$$
\gamma^{0}(v)=\gamma^{0}\left\{1+\Gamma_{2}\left[\left(\frac{v}{v_{p}}\right)^{2}-c\right]\right\}
$$

where $\gamma^{\rho}$ is the thermally averaged pressure broadening coefficient. $v_{p}=\sqrt{\frac{2 k_{B} T}{m_{1}}}$ is the most probable absolute speed of the active molecule of mass $m_{1}$ at a given temperature. The constant $c$ has been fixed to $3 / 2[34,35]$. The parameter $\Gamma_{2}$ is set to 0 if the speed dependence is not taken into account. The speed dependence of the shift can be implemented similarly (we do not have this capability, though). After plugging this expression in the Voigt model and integrating over all possible values for molecular speeds, the real $\left(K_{s}\right)$ and imaginary $\left(L_{s}\right)$ parts of the line shape function become:

$$
\begin{aligned}
& K_{S}(x, y, S)=\frac{2}{\pi} \int_{-\infty}^{\infty} e^{-v^{2}} v \tan ^{-1}\left\{\left[\frac{v+x}{y\left(S\left\{v^{2}-c\right\}+1\right)}\right]^{2}\right\} d v, \\
& L_{S}(x, y, S)=\frac{1}{\pi} \int_{-\infty}^{\infty} e^{-v^{2}} v \ln \left\{\left[\frac{v+x}{y\left(S\left\{v^{2}-c\right\}+1\right)}\right]^{2}+1\right\} d v
\end{aligned}
$$

where $S$ is the line shape.

In our study we have attempted to retrieve the speed dependence parameters for all 45 studied transitions. The remaining weaker transitions had the speed dependence parameter fixed to 0.08. The 0.08 value was chosen based on experimental values of speed dependence parameters retrieved for the $\mathrm{CO}$ molecule in the $2 \leftarrow 0$ band of $\mathrm{CO}$ [38]. To our knowledge there are no reported values for speed dependence coefficients in the $1 \leftarrow 0$ band of CO. In Figure 1 we present a section of the R-branch analyzed with a multispectrum fitting technique using the speed dependent Voigt profile with an asymmetric component to account for weak line mixing. We have ignored the temperature dependence of speed dependence in our study. In a recent study, Lisak et al. [39] have proposed a model for the theoretical temperature dependence of speed dependence. When a larger set of He-broadened CO spectra at higher optical densities and covering many temperatures become available, we will be able to test this model.

\section{THEORETICAL CALCULATIONS}

First, all quantum dynamical calculations were performed, for the work presented in Ref. [24], on the 3-dimensional potential energy surface (PES) for the ${ }^{12} \mathrm{C}^{16} \mathrm{O}-\mathrm{He}$ system proposed by Heijmen et al. [40] in order to provide close-coupling pressure broadening and shift cross sections. These kinetic energy dependent cross sections provided the pressure broadening and shift coefficients at $296 \mathrm{~K}$ given in Ref.[24] for some lines of the fundamental band. Obviously, such calculations allow the determination of the latter parameters at various temperatures (see Eq.(1) of Ref.[24]), what has been done for the present work. Additional calculations were devoted to purely rotational R lines to predict the odd component of the ro-vibrational shifts [24] (see Sect. V). Assuming that the even component (Fig.3b of [24]) has a purely vibrational origin resulting from the difference between the isotropic parts of the PES in the ground and in the first excited vibrational states and is therefore J-independent, we were able to retrieve the shift coefficients in the fundamental (at least at room temperature). A mean 
theoretical value for this even component is about $-0.00048 \mathrm{~cm}^{-1} \mathrm{~atm}^{-1}$ (see Fig.3b of [24]). Moreover, the half width at half maximum (HWHM) is nearly vibrationally independent [29], we thus also present some results of our calculations performed in the vibrational ground state to complete our data analysis.

Second, to provide calculated values of the narrowing parameters we have followed the classical method and relations given in Ref. [20,41]. Assuming that the velocity-changing collisions are independent of the dephasing ones related to the internal states changes, the Dicke narrowing parameter (in $\mathrm{cm}^{-1}$ ) may be fixed to [20,29]:

$$
\beta_{d i f f}^{0}=\frac{v_{p}^{2}}{4 \pi c D_{12}}
$$

In Eq. (9) $v_{p}$ is the most probable speed of the radiative molecule of mass $m_{1}$ and $D_{12}\left(\mathrm{~cm}^{2} \mathrm{~s}^{-1}\right)$ is the binary mass diffusion coefficient $[29,41]$ :

$$
D_{12}=\frac{0.002628}{P \sigma_{12}^{2}} \sqrt{T^{3} \frac{m_{1}+m_{2}}{2 m_{1} m_{2}}} \frac{1}{\Omega_{12}^{(1,1)}\left(T_{12}\right)}
$$

In Eq. (10), $D_{12}$ is expressed in terms of a dimensionless reduced collision integral $\Omega_{12}^{(1,1)}\left(T_{12}\right)$ tabulated in Ref. [41] where $T_{12}$ is a reduced temperature equal ( $\left.k_{\mathrm{B}} T / \varepsilon_{12}\right)$ with $\varepsilon_{12}$ being the well depth of an isotropic potential. Furthermore, $\varepsilon_{12}$ and $\sigma_{12}$ are scaling parameters for the isotropic part ( $V_{\text {iso }}$ ) of the molecular potential. $\sigma_{12}$ (in $\AA$ ) is an equivalent Lennard Jones 6-12 sigma parameter for which $V_{\text {iso }}\left(\sigma_{12}\right.$ )$=0$. The parameters of the isotropic part, in the ground vibrational state of $\mathrm{CO}$, of the potential energy surface (PES) proposed by Heijmen et al. [40] are $\varepsilon_{12} / k_{\mathrm{B}}=22.23 \mathrm{~K}$ and $\sigma_{12}=3.41 \AA$. Note that these parameters are slightly different from those used in Ref. [29]. Other terms in Eq. (10) include the total gas pressure $P$ in atm, $m_{1}$ is the mass of carbon monoxide molecule $(27.995 \mathrm{~g} / \mathrm{mol})$ and $m_{2}$ is the mass of helium (4.0036 g/mol). Combining Eq. (9) and (10) the narrowing parameters, in units of $\mathrm{cm}^{-1} \mathrm{~atm}^{-1}$, at the temperatures of interest may be written [20,29]:

$$
\beta_{\text {diff }}^{0}=0.1664 \sqrt{\frac{2 m_{2}}{m_{1}\left(m_{1}+m_{2}\right)}} \sigma_{12}^{2} \frac{\Omega_{12}^{(1,1)}\left(T_{12}\right)}{\sqrt{T}}
$$

As determined, the narrowing parameter calculated above can be used for comparisons with the optical narrowing parameter retrieved through the line shape analysis. The difference between the later and the one deduced from the mass diffusion coefficient has been discussed in Ref. [42,43] and more recently in Ref. [36]. In Table 1 we present the values of the the narrowing parameter $\beta_{\text {diff }}^{0}$, calculated for temperatures between 10 and $400 \mathrm{~K}$. In the present study we make the approximation that the optical narrowing parameter and the one derived through Eq. (9)-(11) are identical.

\section{COMPARISONS OF RETRIEVED LINE PARAMETERS WITH THEORETICAL AND PUBLISHED VALUES}

As mentioned earlier, we have analyzed our spectra using the Voigt, Rautian, speed dependent Voigt, and Rautian with speed dependence line shapes accounting for line mixing [33]. In the fits 
performed using the Rautian and Rautian with speed dependence model, we have fixed the narrowing parameter due to the Dicke effect to the calculated value at room temperature and presented in Table 1. In our multispectrum analysis, the line intensities were measured line-by-line. Since the main purpose of our work was to study He-broadened line shape parameters we have not performed a detailed analysis of line intensities to the extent of retrieving the band strength, transition dipole moments, and the HermanWallis coefficients. The line parameters for the weak transitions corresponding to the $2 \leftarrow 1$ band were fixed to the HITRAN values [30], except for the He-broadening parameters that were fixed to the values reported in Ref. [25]. We present our retrieval results in a set of tables and figures.

Table 2 presents our results for line positions and line intensities. In Figure 2 we compare our retrieved line positions with those of a multispectrum fit [25] where line positions and line intensities were constrained to known theoretical quantum mechanical expressions, and with the line positions listed in the HITRAN2012 database [30,31]. In both panels the results are presented as a function of the rotational quantum index $m . m=-\mathrm{J}^{\prime \prime}$ for P-branch transitions, $m=\mathrm{J}^{\prime \prime}+1$ for R-branch transitions, where $\mathrm{J}^{\prime \prime}$ is the lower state rotational quantum number. Our present retrieved line positions are about $3.6 \times 10^{-5}$ $\mathrm{cm}^{-1}$ below the line positions obtained in the constrained multispectrum fit [25]. When compared with the line positions reported in the HITRAN2012 database [30], our line positions are about $1.58 \times 10^{-4} \mathrm{~cm}^{-1}$ lower. Panel (B) presents the uncertainties of the retrieved line positions plotted on a logarithmic scale. The uncertainties increase for $|m|<3$ and $|m|>15$.

In Table 2 we present our retrieved line intensities for transitions in the $1 \leftarrow 0$ band in cm/molecule at 296 K. In Fig. 3 we have plotted our measured line intensities as a function of $m$ on linear and logarithmic scales. Our measurements are overlaid with the corresponding line intensities from the HITRAN2012 database [30] in panels (A) and (B) while in panel (D) we present the ratios between the line intensities in the HITRAN2012 database [30] and our measured intensities. Panel C presents the trend in uncertainties in our measured line intensities vs. $|m|$.

The retrieved broadening parameters obtained with the four line shape models and including line mixing are presented in Table 3. The four sets of experimental He-broadening coefficients have been fitted to a semi-empirical power law polynomial expression of the form [44]:

$$
\begin{gathered}
\gamma_{H e}^{0}=a_{0}|m|^{\alpha}, \\
\alpha=a_{1}+a_{2}(\ln |m|)+a_{3}(\ln |m|)^{2}+a_{4}(\ln |m|)^{3}+a_{5}(\ln |m|)^{4} .
\end{gathered}
$$

The expansion coefficients $a_{1}$ to $a_{5}$ are presented in Table 4 for the Voigt, Rautian, speed dependent Voigt, and speed dependent Rautian line shape models. In Table 3 we also include the theoretical values resulting from close coupling calculations for He-broadening coefficients and their temperature dependence exponents. The $\mathrm{CC}$ He-broadening coefficients of some pure rotational lines included in Table 3 highlight the fact that the vibrational dependence of the broadening coefficients is very weak.

Our discussion of He-broadening coefficients and their temperature dependences is completed by Figures 4 and 5. In panel (A) of Fig. 4, we compare our measurement results for He-broadening with the corresponding air-broadening and self-broadening coefficients from HITRAN2012 database [30]. It can be noticed that there is a considerable difference in size between the broadening coefficients for the three types of perturbers. He-broadened line shape parameters are important for many target molecules 
(e.g., $\mathrm{CO}, \mathrm{CH}_{4}$ ) that are trace constituents of planetary atmospheres where $\mathrm{He}$ is a predominant gas. This will ensure proper interpretations of atmospheric remote sensing spectra recorded for these planets.

In panel (B) we display our measured He-broadening coefficients along with previous published data [17, 23-25]. The theoretical and calculated (using Eqs. (12) and (13)) He-broadening coefficients are overlaid in panel $(\mathrm{C})$ of the same figure. Rescaling the experimental line widths measured at $85 \mathrm{~K}$ in Ref. [17] to $79 \mathrm{~K}$ by using the $n$ exponent given in Fig. 5 we have found them to be in good agreement with our observed half widths recorded at $79 \mathrm{~K}$. The differences between our measured He-broadening coefficients and corresponding calculated values using either the power law polynomial fit or the close coupling method, are presented in panels (D) and (E) of Fig. 4. We can observe from Figure 4E that the agreement between observed He-broadening coefficients and those calculated using the close coupling method is very good for all temperatures and $|m|$ values.

Figure 5 presents our results for temperature dependence exponents for He-broadening coefficients of $\mathrm{CO}$ transitions in the $1 \leftarrow 0$ band. Overlaid with these experimental results are the corresponding values from Mantz et al. [25] where the same spectra were used. There are minor differences between our reported values and those of Ref. [25] and we attribute them to the fact that the analysis in Ref. [25] is a constrained multispectrum fit whereas the one in the present study is unconstrained with the inclusion of line mixing. Also displayed are the theoretical temperature dependence exponents for He-broadening. These exponents are obtained using Eq. (1) from theoretical CC He-broadening coefficients computed for five different temperatures (79, 143, 183, 231, and $296 \mathrm{~K})$. These theoretical temperature dependence exponents are all close to 0.54 and slightly different from the classical 0.5 limit value to be used when experimental temperature dependence exponents for broadening coefficients are not available, especially at high temperature for the highest $\mathbf{J}$ values.

Table 5 presents our measurements for He-induced pressure shift coefficients. The listed errors are one standard deviation internal uncertainties. The trends in the measured room temperature Hepressure-shift coefficients as a function of $m$, comparisons with published measurements and theoretical $\mathrm{CC}$ calculations for He-induced pressure shift coefficients are shown in panels (A) and (B) of Figure 6. One important observation is the very small magnitude of He-shift coefficients compared to the corresponding air-shift coefficients. From quantum dynamical calculations performed on the $\mathrm{CO}-\mathrm{He}$ PES we have derived the theoretical He-induced pressure shift coefficients at the five different temperatures mentioned earlier (79, 143, 183, 231, and $296 \mathrm{~K})$. Noticeable are the different trends as a function of $|m|$ for the He-shifts corresponding for the P-branch and R-branch transitions, shown in panel (C). Figure 6D compares the experimental shift coefficients with the close coupling values at different temperatures. The agreement between the two sets of He-pressure shifts gets worse at very low temperatures. However, the calculations predict a very small temperature dependence of the shifts for the temperature range 79-296 K, an experimental behavior that is observed. This is expected because the maximum well depth of the $\mathrm{CO}-\mathrm{He}$ interacting potential $(\sim 20 \mathrm{~K})$ is considerably below the lowest temperature of our gas system $(79 \mathrm{~K})$.

In a study of diatomic molecules, Herman [45] suggested exploring the ability of a given potential energy surface to model the observed broadening and shift coefficients, using the even (e) $m$ and odd (o) $m$ components of the shifts:

$$
\delta_{H e}^{0 e}(m)=\frac{1}{2}\left(\delta_{H e}^{0}(m)+\delta_{H e}^{0}(-m)\right)
$$




$$
\delta_{H e}^{0 o}(m)=\frac{1}{2}\left(\delta_{H e}^{0}(m)-\delta_{H e}^{0}(-m)\right) .
$$

In panels (E) and (F) of Fig. 6 we have plotted the even and odd $m$ components of both measured and theoretical He-pressure shifts at room temperature. The pattern observed in the even $m$ component (Eq. (15) and shown in panel (E)) suggests that it is mostly the difference between the two isotropic components of the interaction potential in the ground and first excited vibrational states that contributes to it (see appendix of [24]). The odd component of the shifts (Eq. (14) and Fig. 6F) is believed to arise from the anisotropic part of the molecular interaction potential (see the discussion in Appendix of Ref. [24]). It essentially represents the shifts of pure rotational lines (see Figs. 3C and 6B of Ref. [24]). The very small magnitude of the shifts and in particular of their even component prove that the differences between the two isotropic components of the interacting potential in $\mathrm{v}=0$ and in $\mathrm{v}=1$ are very small. In other words, that the vibrational motion has a small influence on the shifts for this system. This is due to the triple bond of the $\mathrm{CO}$ molecule and thus of the very small dependence with the intramolecular $\mathrm{CO}$ motion of the vibrationally averaged CO-He PES at least for the very first vibrational quantum numbers. This also explains why the calculated He-broadening coefficients for pure rotational lines and for lines of the fundamental band are so close.

From our experimental and theoretical data one can easily check that the Herman factor [45]:

$$
H(m)=\left(\delta_{H e}^{0}(m)+\delta_{H e}^{0}(-m)\right)\left(\gamma_{H e}^{0}(m)+\gamma_{H e}^{0}(-m)\right)^{3 / 2}
$$

is indeed a function that does not show a dependence in $m$, thus increasing the confidence in both our measurements and theoretical calculations for He-broadening coefficients and He-induced pressure shift coefficients. When plotted, the Herman factor corresponding to our results for He-broadening and Heshifts, shows more scatter than the results from Fig. 8 of Ref. [25], but still no firm dependence on $m$.

In our study we also investigated the temperature dependence of the He-pressure shift coefficients. The expression used in our line parameter retrievals is given in Eq. (3). Table 5 contains our measurements for temperature dependences of He-shift coefficients. Figure 7 panel (A) displays the patterns in the temperature dependence of the pressure-shift coefficients as a function of $m$ for our results and for published results [25]. It can be observed that for positive $m$ values the temperature dependence is close to zero. Even more, panel (B) shows a steeper trend in the temperature dependences as a function of $m$, for P-branch transitions. The temperature dependencies of the He-pressure shifts obtained using close coupling calculations followed a pattern corresponding to a second order dependence in the $\left(T-T_{o}\right)$ term,

$$
\delta_{H e}^{0}(T)=\delta_{H e}^{0}\left(T_{0}\right)+\delta_{1 H e}^{\prime}\left(T-T_{0}\right)+\delta_{2 H e}^{\prime} \frac{\left(T-T_{0}\right)^{2}}{2},
$$

rather than the one described by Eq. (3). Such a pattern has been previously observed for $\mathrm{CO}-\mathrm{H}_{2}$ pressure shifts calculated using a semi-classical approach [46]. The three sets of expansion coefficients for theoretical temperature dependences for the theoretical results are given in Table 6.

As mentioned earlier, in our multispectrum fits we have accounted for weak (Rosenkranz) line mixing effects [33]. The results are presented in Figure 8. In addition, we were unable to retrieve the line mixing for the lines $\mathrm{P}(19)$ to $\mathrm{P}(22)$ and for the lines $\mathrm{R}(20)$ to $\mathrm{R}(22)$. This can be explained by noting that at low temperatures the transitions become weaker due to the Boltzmann distributions. 
We have computed the weak He-broadened line mixing coefficients at room temperature using (i) the semi-empirical Exponential Power Gap (EPG) [47,48] and (ii) the Energy Corrected Sudden (ECS) [47-49] scaling laws. The scaling laws allow us to estimate the diagonal and off-diagonal elements of the relaxation matrix for $\mathrm{CO}$ molecules in a bath of helium molecules. We consider transitions between initial state $i\left(J_{\mathrm{i}}, \mathrm{V}_{\mathrm{i}}=0\right)$ to final state $f\left(J_{\mathrm{f}}, \mathrm{V}_{\mathrm{f}}=1\right)$.

The EPG scaling law offers an expression for the collisional transfer rates $\kappa_{J_{i}{ }^{\prime} \leftarrow J_{i}}(T)$ from the rotational level $J_{i}$ to rotational level $J_{i}$, that depends on three parameters $(a, b$, and $c$ ) that have to be optimized using nonlinear least squares:

$$
\kappa_{J_{i}^{\prime} \leftarrow J_{i}}(T)=a\left[\frac{\mid \Delta E_{i \prime} i}{B}\right]^{-b} \exp \left(\frac{-c\left|\Delta E_{i \prime \prime}\right|}{k_{B} T}\right) .
$$

$\Delta \mathrm{E}_{i^{\prime} i}$ is the energy gap for a downward transition $J_{i^{\prime}} \leftarrow J_{i}$ in $\mathrm{cm}^{-1}, B$ is the rotational constant in the lower energy state.

We used the approximation that the collisional rates between rotational levels in the upper vibrational state are the same as the collisional rates between rotational levels of the lower vibrational state. The He-broadening coefficients are the real components of the diagonal elements of the relaxation matrix $[47,48]$ :

$$
\left\langle\left\langle J_{f} J_{i}|\operatorname{Re}[W]| J_{f} J_{i}\right\rangle\right\rangle=\gamma_{J_{f} \leftarrow J_{i}}=\sum_{J_{i}^{\prime} \neq J_{i}} \kappa_{J_{i}^{\prime} \leftarrow J_{i}}(T) .
$$

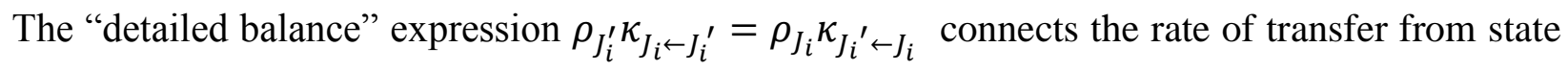
$J_{i}{ }^{\prime}$ to state $J_{i}{ }^{\prime}$ and the population of the rotational level $J_{i}{ }^{\prime}\left(\rho_{J_{i}}{ }^{\prime}\right)$ to the rate of transfer from state $J_{i}$ to state $J_{i}{ }^{\prime}$ and the population of the rotational level $J_{i}\left(\rho_{J_{i}}\right)$. The first order line mixing coefficients, $Y_{i f}^{0}$, due to collisional energy transfer can be calculated using the expression $[33,48]$ :

$$
Y_{J_{f} \leftarrow J_{i}}^{0}=-2 \sum \frac{d_{J_{f}^{\prime} \leftarrow J_{i}^{\prime}}^{\prime}}{d_{J_{f} \leftarrow J_{i}}} \frac{\left\langle\left\langle J^{\prime} f J_{i}^{\prime}|R e[W]| J_{f} J_{i}\right\rangle\right\rangle}{v_{J_{f}^{\prime} \leftarrow J_{i}^{\prime}}-v_{J_{f} \leftarrow J_{i}}}
$$

where $d_{J_{f}^{\prime} \leftarrow J_{i}^{\prime}}$ and $d_{J_{f} \leftarrow J_{i}}$ are dipole moment components corresponding to lines $J_{f}^{\prime} \leftarrow J_{i}^{\prime}$ and $J_{f} \leftarrow J_{i}$, $\left.\left\langle J_{f}^{\prime} J_{i}^{\prime}|R e[W]| J_{f} J_{i}\right\rangle\right\rangle$ are off-diagonal relaxation matrix elements, $v_{J_{f}^{\prime} \leftarrow J_{i}^{\prime}}$ and $v_{J_{f} \leftarrow J_{i}}$ are line positions for the two transitions, in $\mathrm{cm}^{-1}$. In the scaling law approach, the off-diagonal elements of the relaxation matrix are determined as:

$$
\left.\left\langle U_{f}^{\prime} J_{i}^{\prime}|\operatorname{Re}[W]| J_{f} J_{i}\right\rangle\right\rangle=-\eta \kappa_{J_{i}{ }^{\prime} \leftarrow J_{i}}(T),
$$

for $J_{i}^{\prime} \neq J_{i}$ with $\eta=0.55$, value selected because it gave the best agreement between the measured and calculated line mixing coefficients.

Through a nonlinear least squares software written in Matlab we have retrieved the coefficients that would best reproduce both the measured He-broadening coefficients (through Eq. (19)) and the measured line mixing coefficients through Eq. (20). The optimized $a, b, c$ parameters are: 0.015928(4) $\mathrm{cm}^{-1} \mathrm{~atm}^{-1}, 0.244594(6)$, and 1.102272(8) for both P-branch and R-branch transitions. The fitted He-line mixing coefficients are presented in Table 7 and Figure 8. It is encouraging to note that the line mixing coefficients calculated with the EPG law agree well with both the experimental values and with the calculated line mixing coefficients reported in Ref. [14] (through visual inspection of Fig. 6) and in Ref. [17] and references therein.

The Energy Corrected Sudden (ECS) approximation [48,49] is a scaling law obtained by introducing the adiabaticity factor in the Infinite Order Sudden (IOS) approximation [49] for moving 
from initial state $i\left(J_{\mathrm{i}}, \mathrm{v}_{\mathrm{i}}=0\right)$ to final state $f\left(J_{\mathrm{f}}, \mathrm{V}_{\mathrm{f}}=1\right)$. For a simple diatomic molecule as CO interacting with a structureless atom, the off-diagonal elements $\left\langle\left\langle J_{i}^{\prime} J_{f}^{\prime}|W(T)| J_{i} J_{f}\right\rangle\right\rangle$ of the relaxation matrix coupling two downward transitions $J_{f} \leftarrow J_{i}$ and $J_{f}{ }^{\prime} \leftarrow J_{i}^{\prime}$ (where $J_{i}^{\prime}<J_{i}$ ) can be written as:

$$
\begin{gathered}
\left\langle\left\langle J_{i}^{\prime} J_{f}^{\prime}|W(T)| J_{i} J_{f}\right\rangle\right\rangle=\left(2 J_{i}^{\prime}+1\right) \sqrt{\left(2 J_{f}+1\right)\left(2 J_{f}^{\prime}+1\right)} \times \\
\sum_{\text {Leven } \neq 0}\left(\begin{array}{rrr}
J_{i} & L & J_{i}^{\prime} \\
0 & 0 & 0
\end{array}\right)\left(\begin{array}{rrr}
J_{f} & L & J_{f}^{\prime} \\
0 & 0 & 0
\end{array}\right)\left\{\begin{array}{lll}
J_{i} & J_{f} & 1 \\
J_{f}^{\prime} & J_{i}^{\prime} & L
\end{array}\right\} \times(2 L+1) \frac{\Omega_{J>}}{\Omega_{L}} Q_{L}
\end{gathered}
$$

In the above expression, (:::) and $\{:::\}$ are the Wigner $3 \mathrm{~J}$ and $6 \mathrm{~J}$ symbols. The adiabaticity factor $\Omega_{j}$ and basic rate $Q_{L}$ are described by the following expressions [44,45,47]:

$$
\begin{gathered}
\Omega_{J}=\left\{1+\frac{1}{24}\left[\frac{\Delta \omega_{J} d_{c}}{\bar{v}}\right]^{2}\right\}^{-2}, \\
Q_{L}=A\left[\frac{E_{L}}{B}\right]^{-\lambda} \exp \left(-\tau \frac{E_{L}}{k_{B} T}\right) .
\end{gathered}
$$

Here $J<, J>$ are the smallest and largest of a pair $\left(J_{i}, J_{i}{ }^{\prime}\right) ; \Delta \omega_{J}$ is the energy gap between the rotational states $J<, J>$ or $L$ and the following lower rotational state that can be coupled through inelastic collisions. $E_{L}$ is the rotational energy of level $L$ ( $L$ being non-zero); $B$ is the rotational constant; $d_{c}$ is the scaling length that depends on the finite duration of a collision. $\bar{v}$ is the mean relative velocity in $\mathrm{CO}-$ He collisions , $\bar{v}=\sqrt{8 k_{B} T / \pi \mu}$ ( $\mu$ stands for the reduce mass of the molecular system).

The quantities $d_{c}, A, \lambda$, and $\tau$ are marked as the optimized adjustable parameters that have to be determined. A MATLAB code was developed to implement the ECS approach to generate $d_{c}, A, \lambda$, and $\tau$ values by fitting the measured broadening coefficients and making sure that the relations (IV.14) and (IV.15) of Ref. [48] are satisfied. The values $d_{c}, A, \lambda$, and $\tau$ that best reproduce the measured Hebroadening coefficients are $3.5,0.0031,0.72,0.135$. Next, we calculated the line mixing coefficients using the values for $d_{c}(\mathrm{~cm}), A\left(\mathrm{~cm}^{-1} \mathrm{~atm}^{-1}\right), \lambda$, and $\tau$ and Eq. (20). The computed He-line mixing coefficients using the EPG and ECS scaling laws are presented in Table 7 and Figure 8. The line mixing coefficients obtained using the ECS scaling law are more realistic and closer to what we would expect the line mixing coefficients to be.

As mentioned above, we have retrieved the speed dependence parameters (i.e. the $\Gamma_{2}$ terms from Eq. (6)), assumed to be temperature independent, for both the Voigt and Rautian profiles and they are presented in Table 8 as a function of $m$. Due to the smaller optical densities of our spectra, not all the speed dependence parameters are well determined, especially for the weaker high $J$ transitions.

\section{CONCLUSIONS}

In this paper we have re-analyzed the spectra used in Ref. [25] using a line-by-line unconstrained multispectrum approach. The He-broadened line shape parameters have been retrieved using the Voigt, speed dependent Voigt, Rautian, and Rautian with speed dependence line shape models. The best agreement was found for the speed dependent models when line mixing is accounted for. We have used close coupling calculations to compute the He-broadening and He-induced pressure shift coefficients. The retrieved line parameters have been compared with previous measurements and the results of theoretical calculations and were found to agree well at different temperatures. The retrieved weak line mixing coefficients have been compared with results of semi-empirical calculations using the 
Exponential Power Gap law, the Energy Corrected Sudden approximation, and also with published results obtained from quantum dynamical calculations [17] and were found to be in satisfactory agreement.

\section{ACKNOWLEDGEMENTS}

The research carried out at the University of Lethbridge is funded by the Natural Sciences and Engineering Research Council of Canada through the Discovery and CREATE grant programs. The part of the research carried out at the College of William and Mary, Connecticut College and NASA Langley Research Center has been funded by cooperative agreements and contracts with the National Aeronautics and Space Administration. We thank A. Mashwood and N. Islam for their contributions in the early stages of this project. Dr. D. Chris Benner at the College of William and Mary is thanked for allowing us to use his multispectrum fitting software in analysing the data. 


\section{REFERENCES}

[1] Draegert DA, Williams DJ. Collisional broadening of CO absorption lines by foreign gases. J Opt Soc Amer 1968;58:1399-1403.

[2] Duley WW. Interstellar Chemistry, Academic Press; 1984.

[3] Burton WB, Elmegreen BG, Genzel R. The Galactic Interstellar Medium, Springer; 1992.

[4] Tennyson J. Astronomical Spectroscopy, $2^{\text {nd }}$ Edition, World Scientific; 2011.

[5] Hunt RH, Toth RA, Plyler EK. High-resolution determination of widths of self-broadened lines of carbon monoxide. J Chem Phys 1968;49:3909-I2. doi: 10.1063/1.1670699.

[6] Bouanich JP, Haeusler C. Linewidths of carbon monoxide self-broadening and broadened by argon and nitrogen. J Quant Spectrosc Radiat. Transfer 1972;12:695-702. doi:10.1016/00224073(72)90177-X.

[7] Bouanich JP, Brodbeck C. Mesure des largeurs et des deplacements des raies de la bande $0 \rightarrow$ 2 de $\mathrm{CO}$ autoperturbe et perturbe par $\mathrm{N}_{2}, \mathrm{O}_{2}, \mathrm{H}_{2}, \mathrm{HCl}$, $\mathrm{NO}$ et $\mathrm{CO}_{2}$. J Quant Spectrosc Radiat Transfer 1973;13:1-7. doi:10.1016/0022-4073(73)90095-2.

[8] Berger PS, Simon M. Rotational cooling by carbon-monoxide in dark clouds. Astro J 1973;180:L43-L46. doi: 10.1086/181149.

[9] Varanasi P. Measurement of line widths of CO of planetary interest at low temperatures. J Quant Spectrosc Radiat Transfer 1975;15:191-6. doi:10.1016/0022-4073(75)90017-5.

[10] Moskalenko NI. Measurement of intensities and halfwidths of spectral absorption lines of the fundamental 0-1 band of CO. Opt Spectrosc 1975;38:382-4.

[11] Varanasi P, Sarangi S. Measurements of intensities and nitrogen-broadened linewidths in the CO fundamental at low temperatures. J Quant Spectrosc Radiat Transfer 1975;15:473-82. doi:10.1016/0022-4073(75)90066-7.

[12] Nerf Jr RB, Sonnenberg MA. Pressure broadening of the $J=1 \leftarrow 0$ transition of carbon monoxide. J Mol Spectrosc 1975;58:474-8. doi:10.1016/0022-2852(75)90226-X.

[13] Monnanteuil NS, Colmont JM. Pressure broadening of millimeter lines of carbon monoxide. J Mol Spectrosc 1987;126:210-19. doi:10.1016/0022-2852(87)90092-0.

[14] Boissoles J, Boulet C, Robert D, Green S. IOS and ECS line coupling calculation for the COHe system - influence on the vibration-rotation band shapes. J Chem Phys 1987;87:343646. doi:10.1063/1.452988.

[15] Green S, Boissoles J, Boulet C. Accurate collision-induced line-coupling parameters for the fundamental band of $\mathrm{CO}$ in $\mathrm{He}$ : Close coupling and coupled states scattering. J Quant Spectrosc Radiat Transfer 1988;39(1):33-42. doi:10.1016/0022-4073(88)90017-9. 
[16] Mannucci AJ. Heterodyne spectroscopy of carbon-monoxide lines perturbed by hydrogen and helium. J Chem Phys 1991;95:7795-7805. doi:10.1063/1.461310

[17] Thibault F, Boissoles J, Le Doucen R, Farrenq R, Morillon-Chapey M, Boulet C. Line-byline measurements of interference parameters for the 0-1 and 0-2 bands of $\mathrm{CO}$ in $\mathrm{He}$, and comparison with coupled-states calculations. J Chem Phys 1992;97:4623-32. doi:10.1063/1.463865.

[18] Duggan P, Sinclair PM, May AD, Drummond JR. Line-shape analysis of speed-dependent collisional width inhomogeneities in $\mathrm{CO}$ broadened by Xe, $\mathrm{N}_{2}$, and He. Phys Rev A 1995;51:218-24. doi:10.1103/PhysRevA.51.218.

[19] Bouanich JP, Rachet F, Valentin A. Pressure-induced lineshifts in the $1 \leftarrow 0$ band of CO perturbed by He, Ne, Ar, Xe, $\mathrm{O}_{2}$, and $\mathrm{N}_{2}$. J Mol Spectrosc 1996;178:157-64. doi:10.1006/jmsp.1996.0169.

[20] Henry A, Hurtmans D, Margottin-Maclou M, Valentin A. Confinement narrowing and absorber speed dependent broadening effects on $\mathrm{CO}$ lines in the fundamental band perturbed by Xe, Ar, Ne, He and N2. J Quant Spectrosc Radiat Transfer 1996;56:647-71. doi:10.1016/S0022-4073(96)00118-5.

[21] Beaky MM, Goyette TM, De Lucia FC. Pressure broadening and line shift measurements of carbon monoxide in collision with helium from 1 to 600 K. J Chem Phys 1996;105:3994- 4004. doi:10.1063/1.472273.

[22] Thachuk M, Chuaqui CE, Le Roy RJ. Line widths and shifts of very low temperature CO in He: a challenge for theory or experiment ? J Chem Phys 1996;105:4005-14. doi:10.1063/1.472274.

[23] Sinclair PM, Duggan P, Berman R, Drummond JR, May AD. Line broadening in the fundamental band of CO in CO-He and CO-Ar mixtures. J Mol Spectrosc 1998;191:25864. doi:10.1006/jmsp.1998.7628.

[24] Luo C, Wehr R, Drummond JR, May AD, Thibault F, Boissoles J, et al. Shifting and broadening in the fundamental band of $\mathrm{CO}$ highly diluted in He and Ar: A comparison with theory. J Chem Phys 2001;115:2198-206. doi:10.1063/1.1383049.

[25] Mantz AW, Devi VM, Benner DC, Smith MAH, Predoi-Cross A, Dulick M. A multispectrum analysis of widths and shifts in the $2010-2260 \mathrm{~cm}^{-1}$ region of ${ }^{12} \mathrm{C}^{16} \mathrm{O}$ broadened by Helium at temperatures between 80 and 297 K. J Mol Struct 2005;742:99-110. doi: 10.1016/j.molstruc.2004.11.094.

[26] Benner DC, Rinsland CP, Devi VM, Smith MAH, Atkins D. A multispectrum nonlinear least squares fitting technique. J Quant Spectrosc Radiat Transfer 1995;53:705-21. doi:10.1016/0022-4073(95)00015-D.

[27] Mantz AW, Henry A, Valentin A. Stabilized Tunable Diode Laser Measurements of the P(2) Line in the ${ }^{13} \mathrm{CO}$ Fundamental Band Broadened by Helium at Temperatures between 11.5 and 298.6 K. J Mol Spectrosc 2001;207:113-19. doi:10.1006/jmsp.2001.8328.

[28] Henry A, Claveau C, Valentin A, Hurtmans D, Mantz AW. Confinement Narrowing of the $\mathrm{R}(0)$ Line in the ${ }^{13} \mathrm{CO}$ Fundamental Band Broadened by Helium from Room Temperature down to 40 K. J Mol Spectrosc 2002;214:28-34. doi:10.1006/jmsp.2002.8576. 
[29] Thibault F, Mantz AW, Claveau C, Henry A, Valentin A, Hurtmans D. Broadening of the $\mathrm{R}(0)$ and $\mathrm{P}(2)$ lines in the ${ }^{13} \mathrm{CO}$ fundamental by helium atoms from $300 \mathrm{~K}$ down to $12 \mathrm{~K}$ : Measurements and comparison with close-coupling calculations. J Mol Spectrosc 2007;246:118-25. doi:10.1016/j.jms.2007.09.001.

[30] Rothman LS, Gordon IE, Babikov Y, Barbe A, Benner DC, Bernath PF, Birk, et al. The HITRAN 2012 molecular spectroscopic database. J Quant Spectrosc Radiat Transfer 2013;130:4-50. doi:10.1016/j.jqsrt.2013.07.002.

[31] Goorvitch D, Infrared CO line list for the $X^{1} \Sigma^{+}$state, Astrophysical Journal Supplement Series 1994;95:535-52.

[32] Pine AS and Looney JP. $\mathrm{N}_{2}$ and air broadening in the fundamental bands of $\mathrm{HF}$ and $\mathrm{HCl}$. J MoI Spectrosc 1987;122:41-55. doi:10.1016/0022-2852(87)90217-7.

[33] Rosenkranz PW. Shape of 5 mm oxygen band in atmosphere. IEEE Trans Ant Prop 1975; 23(4):498-506. doi:10.1109/TAP.1975.1141119.

[34] Rohart F, Mäder H, Nicolaisen HW. Speed dependence of rotational relaxation induced by foreign gas collisions - studies on $\mathrm{CH}_{3} \mathrm{~F}$ by millimeter-wave coherent transients. J Chem Phys 1994;101:6475-86. doi: 10.1063/1.468342.

[35] Rohart F, Ellendt A, Kaghat F and Mäder H. Self and Polar Foreign Gas Line Broadening and Frequency Shifting of $\mathrm{CH}_{3} \mathrm{~F}$ : Effect of the Speed Dependence Observed by Millimeter Wave Coherent Transients. J Mol Spectrosc 1997;185:222-33. doi:10.1006/jmsp.1997.7395.

[36] Wehr R, Ciurylo R, Vitcu A, Thibault F, Drummond JR, May AD. Dicke-narrowed spectral line shapes of $\mathrm{CO}$ in Ar: Experimental results and a revised interpretation. J Mol Spectrosc 2006; 235:54-68. doi:10.1016/j.jms.2005.10.009.

[37] Devi VM, Benner DC, Brown LR, Miller CE, Toth RA. Line mixing and speed dependence in $\mathrm{CO}_{2}$ at $6348 \mathrm{~cm}^{-1}$ : Positions, intensities, and air- and self-broadening derived with constrained multispectrum analysis. J Mol Spectrosc 2007;242:90-117. doi:10.1016/j.jms.2007.02.018.

[38] Devi VM, Benner DC, Smith MAH, Mantz AW, Sung K, Brown LR, Predoi-Cross A. Spectral line parameters including temperature dependences of self- and air-broadening in the $2 \leftarrow 0$ band of CO at $2.3 \mu \mathrm{m}$. J Quant Spectrosc Radiat Transfer 2012;113:1013-33. doi:10.1016/j.jqsrt.2012.02.010.

[39] Lisak D, Cygan A, Wcislo P, Ciurylo R. Quadratic speed dependence of collisional broadening and shifting for atmospheric applications. J Quant Spectrosc Radiat Transfer 2015;151:43-48. doi:10.1016/j.jqsrt.2014.08.016.

[40] Heijmen TGA, Moszynski R, Wormer PES, van der Avoird A. New He-CO interaction energy surface with vibrational coordinate dependence .1. Ab initio potential and infrared spectrum. J Chem Phys 1997;107:9921-28. doi: 10.1063/1.475290.

[41] Hirschfelder JO, Curtiss ChF, Bird RB. Molecular Theory of Gases and Liquids, New York: Wiley; 1967.

[42] Corey GC, McCourt FRW. Dicke narrowing and collisional broadening of spectral-lines in dilute molecular gases. J Chem Phys 1984;81:2318-29. doi: 10.1063/1.447930. 
[43] Blackmore R. A modified Boltzmann kinetic equation for line shape functions. J Chem Phys 1987;87:791-800. doi: 10.1063/1.453286

[44] Predoi-Cross A, Sinclair PM, Luo C, Drummond JR, May AD, Line broadening and the temperature exponent of the fundamental band in CO- $\mathrm{N}_{2}$ mixtures. J Mol Spectrosc 1999;198(2):291303. doi:10.1006/jmsp. 1999.7940

[45] Herman R, Impact theory of noble-gas-broadened HCL vibration-rotation lines. Phys Rev 1963;132:262-75. doi: 10.1103/PhysRev.132.262.

[46] Bouanich JP, Predoi-Cross A, Theoretical calculations for line-broadening and pressureshifting in the fundamental and first two overtone bands of CO- $\mathrm{H}_{2}$. J Mol Struct 2005;742 (1-3):183-90. doi:10.1016/j.molstruc.2005.01.004.

[47] Predoi-Cross A, Bouanich JP, Benner DC, Drummond JR, May AD. Broadening, shifting, and line asymmetries in the $2 \leftarrow 0$ band of $\mathrm{CO}$ and $\mathrm{CO}-\mathrm{N}_{2}$ : Experimental results and theoretical calculations. J Chem Phys 2000;113(1):158-68. doi: 10.1063/1.481783.

[48] Hartmann JM, Robert D, Boulet C. Collisional effects on molecular spectra. Elsevier Science 2008.

[49] Brunner TA, Prichard D. In: K.P. Lawley (Ed.), Advances in chemical physics, volume 50: Dynamics of the excited state. John Wiley and Sons Ltd; 1982. 


\section{List of Tables}

Table 1. Values of effective narrowing coefficients $\left(\beta_{\text {diff }}^{0}\right)$ calculated for temperatures between $10 \mathrm{~K}$ and $400 \mathrm{~K}$.

Table 2. Line positions retrieved using the Voigt profile and intensities multiplied by a factor of $10^{20}$ in $\mathrm{cm}^{-1} /\left(\right.$ molecule $\left.\times \mathrm{cm}^{-2}\right)$ at $296 \mathrm{~K}$.

Table 3. He-broadening coefficients in $\mathrm{cm}^{-1} \mathrm{~atm}^{-1}$ at $296 \mathrm{~K}$ and their temperature dependence exponents (unitless) obtained using the Voigt, Rautian, speed dependent Voigt (SD-Voigt), and Rautian with speed dependence (SD-Rautian) line shape models. We accounted for line mixing in all our multispectrum fits. Also included are the room temperature $(296 \mathrm{~K})$ theoretical He-broadening parameters described in section IV of the manuscript and their temperature dependences.

Table 4. Power law polynomial fit room temperature coefficients of measured He-broadening coefficients to the expression presented in Eq. (12). The headings for columns two to five correspond to the line shape model used to retrieve the experimental He-broadening coefficients.

Table 5. CO-He pressure induced shift coefficients $\left(\mathrm{cm}^{-1} \mathrm{~atm}^{-1}\right.$ at $\left.296 \mathrm{~K}\right)$ and their temperature dependences in $\mathrm{cm}^{-1}$ atm $^{-1} \mathrm{~K}^{-1}$ from Eq. (3) obtained using the Voigt, Rautian, speed dependent Voigt (SD-Voigt), and Rautian with speed dependence (SD-Rautian) line shape models. The heading for each column contains the name of the line shape used in the retrievals. All fits included line mixing.

Table 6. Theoretical close coupling He-broadened pressure-shift coefficients $\left(\mathrm{cm}^{-1} \mathrm{~atm}^{-1}\right)$ at $296 \mathrm{~K}$ and their temperature dependences as described by Eq. (17).

Table 7. Comparisons of measured line mixing coefficients ( $\mathrm{atm}^{-1}$ at $296 \mathrm{~K}$ ) for $\mathrm{CO}$ in $\mathrm{He}$ using Rosenkranz approximation to those computed with the Exponential Power Gap (EPG) and Energy Corrected Sudden (ECS) scaling laws.

Table 8. Retrieved speed dependence parameters $\Gamma_{2}$ (no units) for the $\mathrm{P}$ - and R-branch transitions in the $1 \leftarrow 0$ band of $\mathrm{CO}$ in helium. Weak line mixing was included in the fits. 


\section{List of figures}

Figure 1. A section of the 19 overlaid spectra analyzed applying the multispectrum nonlinear least squares fitting technique using the speed dependent Voigt profile with weak line mixing (A). Overlaid observed minus calculated fit residuals (B)

Figure 2. (A) Differences between the line positions retrieved in the present study, in HITRAN2012 database [30] and the results of the constrained multispectrum fit analysis reported in Ref. [25]. (B) Uncertainties of the retrieved line positions plotted on a logarithmic scale.

Figure 3. Retrieved line intensities for transitions in the $1 \leftarrow 0$ band plotted as a function of $m$ ( $m=-J^{\prime \prime}$ and $J^{\prime \prime}+1$ for the P- and R-branch transitions, respectively) on a linear scale (A) and on a logarithmic scale (B). Present measurements are compared with the corresponding line intensities from the HITRAN2012 database [30]. (C) Uncertainties in our fitted line intensities given as percentage of measured line intensities. (D) Ratios between our retrieved line intensities and the line intensities in the HITRAN 2012 database [30] at $296 \mathrm{~K}$. Here, we label line parameters retrieved using the speed dependent Voigt line shape model as "SD-Voigt". Similarly, line parameters retrieved using the speed dependent Rautian line shape model are labelled as "SD-Rautian".

Figure 4. Retrieved CO-He broadening coefficients in $\mathrm{cm}^{-1} \mathrm{~atm}^{-1}$ at $296 \mathrm{~K}$ obtained using the Voigt, Rautian, speed dependent Voigt (SD-Voigt), and speed dependent Rautian (SD-Rautian) line shapes including line mixing (LM). Overlaid with our measurement results are: the air-broadened and selfbroadened half width coefficients from HITRAN2012 [30] (A), previously published measurements of CO-He broadening coefficients (B). In panel (C) Measured CO-He broadening coefficients as a function of the rotational quantum index $m$ overlaid with theoretical He-broadening coefficients discussed in section IV and values fitted using power law polynomial fit and in panel (D) Differences between our measured He-broadening coefficients at room temperature $(296 \mathrm{~K})$ and those calculated using the empirical power law polynomial fit. Overlaid to them is the difference between our measured Hebroadening coefficients obtained using the Voigt profile and the corresponding theoretical (CC) values. (E) Experimental and theoretical (CC) broadening coefficients at different temperatures.

Figure 5. Temperature dependence exponents (unitless) for He-broadening coefficients of CO transitions in the fundamental band.

Figure 6. (A) Measured CO-He induced pressure-shift coefficients $\left(\mathrm{cm}^{-1} \mathrm{~atm}^{-1}\right.$ at $\left.296 \mathrm{~K}\right)$ obtained using the Voigt, Rautian, speed dependent Voigt (SD-Voigt), and speed dependent Rautian (SD-Rautian) shape models with line mixing. Overlaid with our retrieved results are: the air-induced pressure-shift coefficients from HITRAN2012 [30] (A), previously published measurements and the calculated COHe pressure-shift coefficients (B), retrieved He-pressure shift coefficients plotted against $|m|$, plotted separately for the R- and P-branch transitions (C). In panel (D) we have plotted the experimental Heshift coefficients and the close coupling (CC) values at different temperatures. The CO-He pressure shift coefficients are separated for even (panel (E) and Eq. (14)) and odd (panel (F) and Eq. (15)) $\mathrm{m}$ components, plotted as a function of $m$. 
Figure 7. (A) Retrieved temperature dependences for He-pressure shift coefficients $\left(\mathrm{cm}^{-1} \mathrm{~atm}^{-1} \mathrm{~K}^{-1}\right.$ at $296 \mathrm{~K}$ ) overlaid with the results from Ref. [25] plotted as a function of $m$. (B) Measured temperature dependence coefficients for the R- and P-branch transitions and plotted as a function of $|m|$.

Figure 8. (A) Measured, calculated and previously published [17] CO-He collisional line mixing coefficients (in atm ${ }^{-1}$ at $296 \mathrm{~K}$ ) for transitions in the $1 \leftarrow 0$ band of CO. 
Table 1.

\begin{tabular}{|c|c|}
\hline Temperature (K) & $\beta_{\text {diff }}^{o}\left(\mathrm{~cm}^{-1} \mathbf{a t m}^{-1}\right)$ \\
\hline 10 & 0.127 \\
\hline 15 & 0.083 \\
\hline 20 & 0.062 \\
\hline 25 & 0.050 \\
\hline 30 & 0.042 \\
\hline 35 & 0.036 \\
\hline 40 & 0.032 \\
\hline 45 & 0.029 \\
\hline 50 & 0.027 \\
\hline 55 & 0.025 \\
\hline 60 & 0.023 \\
\hline 65 & 0.022 \\
\hline 70 & 0.021 \\
\hline 75 & 0.020 \\
\hline 80 & 0.019 \\
\hline 85 & 0.018 \\
\hline 90 & 0.017 \\
\hline 95 & 0.016 \\
\hline 100 & 0.016 \\
\hline 105 & 0.015 \\
\hline 110 & 0.015 \\
\hline 115 & 0.014 \\
\hline 120 & 0.014 \\
\hline 125 & 0.014 \\
\hline 130 & 0.013 \\
\hline 135 & 0.013 \\
\hline 140 & 0.013 \\
\hline 145 & 0.012 \\
\hline 150 & 0.012 \\
\hline 155 & 0.012 \\
\hline 160 & 0.011 \\
\hline 165 & 0.011 \\
\hline 170 & 0.011 \\
\hline 175 & 0.011 \\
\hline 180 & 0.011 \\
\hline 185 & 0.010 \\
\hline 190 & 0.010 \\
\hline 195 & 0.010 \\
\hline 200 & 0.010 \\
\hline 205 & 0.010 \\
\hline 210 & 0.009 \\
\hline 215 & 0.009 \\
\hline 220 & 0.009 \\
\hline 225 & 0.009 \\
\hline 230 & 0.009 \\
\hline 235 & 0.009 \\
\hline 240 & 0.009 \\
\hline 245 & 0.009 \\
\hline 250 & 0.008 \\
\hline 255 & 0.008 \\
\hline 260 & 0.008 \\
\hline
\end{tabular}




\begin{tabular}{ll}
265 & 0.008 \\
270 & 0.008 \\
275 & 0.008 \\
280 & 0.008 \\
285 & 0.008 \\
290 & 0.008 \\
297 & 0.008 \\
295 & 0.008 \\
300 & 0.008 \\
305 & 0.007 \\
310 & 0.007 \\
315 & 0.007 \\
320 & 0.007 \\
325 & 0.007 \\
330 & 0.007 \\
335 & 0.007 \\
340 & 0.007 \\
345 & 0.007 \\
350 & 0.007 \\
355 & 0.007 \\
360 & 0.007 \\
365 & 0.007 \\
370 & 0.007 \\
375 & 0.007 \\
380 & 0.006 \\
385 & 0.006 \\
390 & 0.006 \\
395 & 0.006 \\
400 & 0.006 \\
\hline
\end{tabular}


Table 2.

\begin{tabular}{|c|c|c|c|c|c|}
\hline \multirow{2}{*}{ Label } & \multirow{2}{*}{$\begin{array}{c}\text { Line } \\
\text { position } \\
\left(\mathrm{cm}^{-1}\right)\end{array}$} & \multicolumn{4}{|c|}{ Line intensities $\left(10^{-20} \mathrm{~cm}^{-1} /\left(\right.\right.$ molecule $\left.\left.\cdot \mathrm{cm}^{-2}\right)\right)$} \\
\hline & & Voigt & Rautian & SD-Voigt & SD-Rautian \\
\hline $\mathrm{P}(22)$ & $2050.854230(41)$ & $1.746(4)$ & $1.747(4)$ & $1.744(5)$ & $1.744(5)$ \\
\hline $\mathrm{P}(21)$ & $2055.400523(30)$ & $2.501(4)$ & $2.501(4)$ & $2.501(5)$ & $2.501(5)$ \\
\hline $\mathrm{P}(20)$ & 2059.914818(22) & $3.540(4)$ & $3.541(4)$ & $3.546(5)$ & $3.545(5)$ \\
\hline $\mathrm{P}(19)$ & 2064.397136(17) & $4.885(4)$ & $4.887(4)$ & $4.916(5)$ & $4.921(5)$ \\
\hline $\mathrm{P}(18)$ & 2068.847101(13) & $6.596(4)$ & $6.597(4)$ & $6.606(5)$ & $6.607(5)$ \\
\hline $\mathrm{P}(17)$ & 2073.264734(11) & $8.717(4)$ & $8.718(4)$ & $8.715(4)$ & $8.713(5)$ \\
\hline $\mathrm{P}(16)$ & 2077.649897(9) & $11.273(4)$ & $11.275(4)$ & $11.271(4)$ & $11.269(5)$ \\
\hline $\mathrm{P}(15)$ & 2082.002395(7) & $14.295(4)$ & $14.297(4)$ & $14.306(5)$ & $14.306(5)$ \\
\hline $\mathrm{P}(14)$ & $2086.322093(6)$ & $17.598(4)$ & $17.601(4)$ & $17.598(5)$ & $17.596(5)$ \\
\hline $\mathrm{P}(13)$ & $2090.608820(5)$ & $21.280(4)$ & $21.284(4)$ & $21.304(5)$ & $21.305(5)$ \\
\hline $\mathrm{P}(12)$ & $2094.862460(5)$ & $25.074(5)$ & $25.078(5)$ & $25.103(5)$ & $25.105(5)$ \\
\hline $\mathrm{P}(11)$ & 2099.082891(4) & $28.743(5)$ & $28.748(5)$ & $28.754(6)$ & $28.752(6)$ \\
\hline $\mathrm{P}(10)$ & 2103.269861(4) & $32.124(6)$ & $32.131(6)$ & $32.189(7)$ & $32.194(7)$ \\
\hline $\mathrm{P}(9)$ & $2107.423347(4)$ & $34.768(7)$ & $34.777(7)$ & 34.728(9) & $34.724(9)$ \\
\hline $\mathrm{P}(8)$ & $2111.543144(4)$ & 36.588(9) & 36.599(9) & $36.599(12)$ & $36.601(12)$ \\
\hline $\mathrm{P}(7)$ & $2115.629086(4)$ & $37.187(11)$ & $37.202(11)$ & $37.16(15)$ & $37.155(15)$ \\
\hline $\mathrm{P}(6)$ & 2119.681093(4) & $36.383(11)$ & $36.396(11)$ & $36.349(15)$ & $36.344(15)$ \\
\hline $\mathrm{P}(5)$ & 2123.698944(4) & $33.984(10)$ & $33.997(10)$ & $34.036(14)$ & $34.044(14)$ \\
\hline $\mathrm{P}(4)$ & 2127.682537(4) & 29.944(9) & 29.954(9) & 29.935(11) & 29.931(11) \\
\hline $\mathrm{P}(3)$ & $2131.631713(4)$ & $24.328(6)$ & $24.334(6)$ & $24.406(8)$ & $24.410(8)$ \\
\hline $\mathrm{P}(2)$ & $2135.546316(4)$ & $17.212(4)$ & $17.216(4)$ & $17.248(4)$ & $17.251(4)$ \\
\hline $\mathrm{P}(1)$ & 2139.426212(5) & 8.933(2) & $8.934(2)$ & $8.942(2)$ & $8.943(2)$ \\
\hline $\mathrm{R}(0)$ & $2147.081274(5)$ & $9.127(2)$ & $9.128(2)$ & $9.134(2)$ & $9.135(2)$ \\
\hline $\mathrm{R}(1)$ & $2150.856162(4)$ & $17.983(4)$ & $17.987(4)$ & $18.034(5)$ & $18.039(5)$ \\
\hline $\mathrm{R}(2)$ & $2154.595695(4)$ & $25.953(7)$ & $25.962(7)$ & 26.093(9) & 26.102(9) \\
\hline $\mathrm{R}(3)$ & $2158.299826(4)$ & $32.665(10)$ & $32.678(10)$ & 32.671(13) & $32.675(13)$ \\
\hline $\mathrm{R}(4)$ & $2161.968374(4)$ & $37.857(13)$ & $37.872(13)$ & $37.888(18)$ & 37.897(18) \\
\hline $\mathrm{R}(5)$ & $2165.601163(4)$ & 41.397(15) & $41.416(15)$ & $41.403(22)$ & $41.415(21)$ \\
\hline $\mathrm{R}(6)$ & 2169.198089(4) & $43.248(16)$ & $43.266(16)$ & $43.128(23)$ & $43.119(23)$ \\
\hline $\mathrm{R}(7)$ & 2172.758969(4) & $43.440(15)$ & $43.457(15)$ & $43.347(22)$ & $43.339(21)$ \\
\hline $\mathrm{R}(8)$ & $2176.283651(4)$ & $42.196(12)$ & $42.212(12)$ & 42.131(17) & $42.129(16)$ \\
\hline $\mathrm{R}(9)$ & $2179.772029(4)$ & 39.824(9) & 39.835(9) & 39.786(12) & 39.782(12) \\
\hline $\mathrm{R}(10)$ & 2183.223924(4) & $36.565(8)$ & $36.574(8)$ & 36.579(9) & $36.579(9)$ \\
\hline $\mathrm{R}(11)$ & 2186.639172(4) & $32.633(6)$ & $32.640(6)$ & $32.668(7)$ & $32.671(7)$ \\
\hline $\mathrm{R}(12)$ & 2190.017699(5) & $28.377(5)$ & $28.382(5)$ & $28.400(6)$ & $28.401(6)$ \\
\hline $\mathrm{R}(13)$ & 2193.359297(5) & $24.072(5)$ & $24.077(5)$ & $24.104(6)$ & $24.107(6)$ \\
\hline $\mathrm{R}(14)$ & 2196.663829(6) & $19.952(4)$ & $19.956(4)$ & $19.983(5)$ & $19.985(5)$ \\
\hline $\mathrm{R}(15)$ & 2199.931166(7) & $16.123(4)$ & $16.126(4)$ & $16.136(5)$ & $16.136(5)$ \\
\hline $\mathrm{R}(16)$ & 2203.161156(8) & $12.749(4)$ & $12.751(4)$ & $12.753(5)$ & $12.753(5)$ \\
\hline $\mathrm{R}(17)$ & $2206.353648(10)$ & $9.879(4)$ & $9.881(4)$ & $9.892(5)$ & $9.893(5)$ \\
\hline $\mathrm{R}(18)$ & $2209.508488(13)$ & $7.476(4)$ & $7.478(4)$ & $7.485(5)$ & $7.484(5)$ \\
\hline
\end{tabular}




\begin{tabular}{llllll}
$\mathrm{R}(19)$ & $2212.625520(16)$ & $5.526(4)$ & $5.527(4)$ & $5.533(5)$ & $5.532(5)$ \\
$\mathrm{R}(20)$ & $2215.704655(21)$ & $4.026(4)$ & $4.026(4)$ & $4.041(5)$ & $4.040(5)$ \\
$\mathrm{R}(21)$ & $2218.745697(28)$ & $2.865(4)$ & $2.865(4)$ & $2.878(5)$ & $2.878(5)$ \\
$\mathrm{R}(22)$ & $2221.748400(38)$ & $1.992(4)$ & $1.992(4)$ & $1.987(5)$ & $1.987(5)$ \\
\hline \hline
\end{tabular}




\begin{tabular}{|c|c|c|c|c|c|c|c|c|c|c|}
\hline \multirow{2}{*}{ Line } & \multicolumn{5}{|c|}{ He-Broadening Coefficients } & \multicolumn{5}{|c|}{ Temperature Dependence Exponents } \\
\hline & Voigt & SD-Voigt & Rautian & SD-Rautian & Theory (CC) & Voigt & SDV & Rautian & SD-Rautian & Theory (CC) \\
\hline P(22) & 0.04556(16) & "0.04537(32) & 0.04559(16) & $0.04531(34)$ & & $0.499(22)$ & $0.499(22)$ & $0.499(22)$ & $0.499(22)$ & \\
\hline $\mathrm{P}(21)$ & $0.04577(12)$ & $0.04577(23)$ & $0.04581(12)$ & $0.0457(24)$ & & $0.550(14)$ & $0.550(14)$ & $0.550(14)$ & $0.550(14)$ & \\
\hline$P(20)$ & $0.04615(9)$ & $0.04642(18)$ & $0.04619(9)$ & $0.0464(20)$ & & $0.563(9)$ & $0.563(9)$ & $0.561(9)$ & $0.563(9)$ & \\
\hline $\mathrm{P}(19)$ & $0.04644(7)$ & $0.04779(15)$ & $0.04648(6)$ & $0.04809(16)$ & & $0.555(6)$ & $0.557(6)$ & $0.555(6)$ & $0.558(6)$ & \\
\hline $\mathrm{P}(18)$ & $0.04645(5)$ & $0.04677(10)$ & $0.04648(5)$ & $0.04679(11)$ & & $0.557(4)$ & $0.558(4)$ & $0.557(4)$ & $0.558(4)$ & \\
\hline $\mathrm{P}(17)$ & $0.04640(4)$ & $0.04637(7)$ & $0.04643(4)$ & $0.04631(7)$ & & $0.562(3)$ & $0.561(3)$ & $0.561(3)$ & $0.561(3)$ & \\
\hline $\mathrm{P}(16)$ & $0.04658(3)$ & $0.04653(5)$ & $0.04661(3)$ & $0.04648(6)$ & & $0.560(2)$ & $0.560(2)$ & $0.560(2)$ & $0.560(2)$ & \\
\hline $\mathrm{P}(15)$ & $0.04683(3)$ & $0.04698(5)$ & $0.04686(3)$ & $0.04698(5)$ & & $0.573(2)$ & $0.573(2)$ & $0.572(2)$ & $0.573(2)$ & \\
\hline $\mathrm{P}(14)$ & $0.04672(3)$ & $0.04670(4)$ & $0.04673(3)$ & $0.04667(4)$ & & $0.570(1)$ & $0.57(1)$ & $0.570(1)$ & $0.570(1)$ & \\
\hline $\mathrm{P}(13)$ & $0.04698(3)$ & $0.04714(3)$ & $0.04699(3)$ & $0.04715(3)$ & & $0.570(1)$ & $0.571(1)$ & $0.569(1)$ & $0.571(1)$ & \\
\hline $\mathrm{P}(12)$ & $0.04705(3)$ & $0.04719(3)$ & $0.04706(3)$ & $0.04720(3)$ & & $0.571(1)$ & $0.573(1)$ & $0.571(1)$ & $0.573(1)$ & \\
\hline $\mathrm{P}(11)$ & $0.04675(2)$ & $0.04678(3)$ & $0.04676(2)$ & $0.04676(3)$ & 0.0460 & $0.576(1)$ & $0.576(1)$ & $0.576(1)$ & $0.576(1)$ & 0.54 \\
\hline $\mathrm{P}(10)$ & $0.04685(2)$ & $0.04696(3)$ & $0.04685(2)$ & $0.04697(3)$ & 0.0458 & $0.584(1)$ & $0.586(1)$ & $0.584(1)$ & $0.586(1)$ & 0.54 \\
\hline $\mathrm{P}(9)$ & $0.04654(2)$ & $0.04652(2)$ & $0.04653(2)$ & $0.04650(2)$ & 0.0458 & $0.575(1)$ & $0.575(1)$ & $0.575(1)$ & $0.574(1)$ & 0.54 \\
\hline $\mathrm{P}(8)$ & $0.04671(2)$ & $0.04671(2)$ & $0.04670(2)$ & $0.04670(2)$ & 0.0457 & $0.570(1)$ & $0.57(1)$ & $0.570(1)$ & $0.570(1)$ & 0.54 \\
\hline $\mathrm{P}(7)$ & $0.04653(2)$ & $0.04654(2)$ & $0.04651(2)$ & $0.04652(2)$ & & $0.560(1)$ & $0.560(1)$ & $0.560(1)$ & $0.560(1)$ & \\
\hline $\mathrm{P}(6)$ & $0.04647(2)$ & $0.04648(2)$ & $0.04646(2)$ & $0.04647(2)$ & & $0.549(1)$ & $0.550(1)$ & $0.549(1)$ & $0.550(1)$ & \\
\hline$P(5)$ & $0.04663(2)$ & $0.04664(2)$ & $0.04662(2)$ & $0.04662(2)$ & & $0.533(1)$ & $0.533(1)$ & $0.533(1)$ & $0.532(1)$ & \\
\hline $\mathrm{P}(4)$ & $0.04633(2)$ & $0.04633(2)$ & $0.04632(2)$ & $0.04631(2)$ & & $0.522(1)$ & $0.522(1)$ & $0.522(1)$ & $0.522(1)$ & \\
\hline $\mathrm{P}(3)$ & $0.04673(2)$ & $0.04690(3)$ & $0.04673(2)$ & $0.04691(3)$ & 0.0456 & $0.511(1)$ & $0.507(1)$ & $0.511(1)$ & $0.507(1)$ & 0.54 \\
\hline$P(2)$ & $0.04715(2)$ & $0.04739(3)$ & $0.04716(2)$ & $0.04740(3)$ & 0.0461 & $0.506(1)$ & $0.501(1)$ & $0.505(1)$ & $0.501(1)$ & 0.54 \\
\hline $\mathrm{P}(1)$ & $0.04878(3)$ & $0.04900(4)$ & $0.04880(3)$ & $0.04901(4)$ & 0.0481 & $0.516(1)$ & $0.513(1)$ & $0.516(1)$ & $0.513(1)$ & 0.53 \\
\hline $\mathrm{R}(0)$ & $0.04880(3)$ & $0.04898(4)$ & $0.04882(3)$ & $0.04898(4)$ & 0.0481 & $0.514(1)$ & $0.511(1)$ & $0.513(1)$ & $0.511(1)$ & 0.53 \\
\hline $\mathrm{R}(1)$ & $0.04718(2)$ & $0.04749(3)$ & $0.04719(2)$ & $0.04752(3)$ & 0.0461 & $0.509(1)$ & $0.504(1)$ & $0.509(1)$ & $0.503(1)$ & 0.54 \\
\hline $\mathrm{R}(2)$ & $0.04703(2)$ & $0.04729(3)$ & $0.04702(2)$ & $0.04731(3)$ & 0.0457 & $0.509(1)$ & $0.502(1)$ & $0.509(1)$ & $0.502(1)$ & 0.54 \\
\hline $\mathrm{R}(3)$ & $0.04663(2)$ & $0.04663(2)$ & $0.04661(2)$ & $0.04662(2)$ & $0.0450^{(\mathrm{a})}$ & $0.519(1)$ & $0.519(1)$ & $0.519(1)$ & $0.519(1)$ & \\
\hline $\mathrm{R}(4)$ & $0.04681(3)$ & $0.04680(3)$ & $0.04679(3)$ & $0.04679(3)$ & $0.0450^{(a)}$ & $0.531(1)$ & $0.531(1)$ & $0.531(1)$ & $0.531(1)$ & \\
\hline $\mathrm{R}(5)$ & $0.04688(3)$ & $0.04687(3)$ & $0.04685(3)$ & $0.04685(3)$ & $0.0450^{(\mathrm{a})}$ & $0.543(1)$ & $0.543(1)$ & $0.543(1)$ & $0.543(1)$ & \\
\hline $\mathrm{R}(6)$ & $0.04679(3)$ & $0.04686(3)$ & $0.04677(3)$ & $0.04684(3)$ & $0.0450^{(a)}$ & $0.557(1)$ & $0.558(1)$ & $0.557(1)$ & $0.558(1)$ & \\
\hline $\mathrm{R}(7)$ & $0.04706(3)$ & $0.04710(3)$ & $0.04704(3)$ & $0.04709(3)$ & 0.0456 & $0.567(1)$ & $0.568(1)$ & $0.567(1)$ & $0.568(1)$ & 0.54 \\
\hline
\end{tabular}




\begin{tabular}{|c|c|c|c|c|c|c|c|c|c|c|}
\hline $\mathrm{R}(8)$ & $0.04716(3)$ & $0.04718(3)$ & $0.04714(3)$ & $0.04716(3)$ & 0.0457 & $0.575(1)$ & $0.575(1)$ & $0.575(1)$ & $0.575(1)$ & 0.54 \\
\hline $\mathrm{R}(9)$ & $0.04709(2)$ & $0.04709(2)$ & $0.04707(2)$ & $0.04706(2)$ & 0.0459 & $0.58(1)$ & $0.579(1)$ & $0.580(1)$ & $0.579(1)$ & 0.54 \\
\hline $\mathrm{R}(10)$ & $0.04707(2)$ & $0.04707(3)$ & $0.04706(2)$ & $0.04706(3)$ & 0.0459 & $0.582(1)$ & $0.583(1)$ & $0.582(1)$ & $0.582(1)$ & 0.54 \\
\hline $\mathrm{R}(11)$ & $0.04720(2)$ & $0.04726(3)$ & $0.04720(2)$ & $0.04726(3)$ & & $0.577(1)$ & $0.578(1)$ & $0.577(1)$ & $0.578(1)$ & \\
\hline $\mathrm{R}(12)$ & $0.04712(3)$ & $0.04719(3)$ & $0.04712(3)$ & $0.04719(3)$ & & $0.574(1)$ & $0.576(1)$ & $0.575(1)$ & $0.576(1)$ & \\
\hline $\mathrm{R}(13)$ & $0.04718(3)$ & $0.04737(3)$ & $0.04719(3)$ & $0.04738(3)$ & & $0.565(1)$ & $0.568(1)$ & $0.565(1)$ & $0.568(1)$ & \\
\hline $\mathrm{R}(14)$ & $0.04700(3)$ & $0.04725(4)$ & $0.04701(3)$ & $0.04726(4)$ & $0.0462^{(a)}$ & $0.568(1)$ & $0.570(1)$ & $0.568(1)$ & $0.570(1)$ & \\
\hline $\mathrm{R}(15)$ & $0.04671(3)$ & $0.04684(4)$ & $0.04674(3)$ & $0.04683(4)$ & & $0.573(2)$ & $0.574(2)$ & $0.573(2)$ & $0.573(2)$ & \\
\hline $\mathrm{R}(16)$ & $0.04651(3)$ & $0.04655(5)$ & $0.04653(3)$ & $0.04653(5)$ & & $0.572(2)$ & $0.572(2)$ & $0.572(2)$ & $0.572(2)$ & \\
\hline $\mathrm{R}(17)$ & $0.04655(4)$ & $0.04681(7)$ & $0.04657(4)$ & $0.04682(7)$ & & $0.556(3)$ & $0.556(3)$ & $0.555(3)$ & $0.556(3)$ & \\
\hline $\mathrm{R}(18)$ & $0.04642(5)$ & 0.04663(9) & $0.04645(5)$ & $0.04660(9)$ & & $0.550(4)$ & $0.550(4)$ & $0.550(4)$ & $0.550(4)$ & \\
\hline $\mathrm{R}(19)$ & $0.04620(6)$ & $0.04644(12)$ & $0.04623(6)$ & $0.04642(13)$ & & $0.541(6)$ & $0.541(6)$ & $0.541(6)$ & $0.541(6)$ & \\
\hline $\mathrm{R}(20)$ & $0.04594(8)$ & $0.04672(19)$ & $0.04594(8)$ & $0.04671(19)$ & $0.0454^{(a)}$ & $0.556(8)$ & $0.557(8)$ & $0.556(8)$ & $0.557(8)$ & \\
\hline $\mathrm{R}(21)$ & $0.04588(11)$ & $0.04692(27)$ & $0.04589(11)$ & $0.04691(27)$ & & $0.533(13)$ & $0.535(13)$ & $0.533(13)$ & $0.535(13)$ & \\
\hline $\mathrm{R}(22)$ & $0.04569(15)$ & $0.04509(31)$ & $0.04569(15)$ & $0.04510(31)$ & & $0.481(20)$ & $0.480(20)$ & $0.481(20)$ & $0.480(20)$ & \\
\hline
\end{tabular}

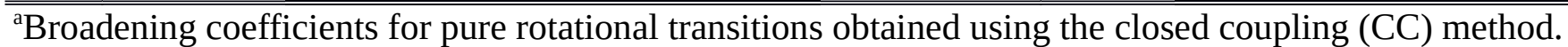


Table 4.

\begin{tabular}{ccccc}
\hline \hline Coefficients & Voigt & SDV & Rautian $^{\mathbf{a}}$ & SD-Rautian $^{\mathbf{a}}$ \\
\hline \hline $\boldsymbol{a}_{\boldsymbol{0}}$ & -3.020322 & -3.016192 & -3.019905 & -3.016102 \\
$\boldsymbol{a}_{\mathbf{1}}$ & 0.063473 & -0.059410 & -0.065503 & -0.055430 \\
$\boldsymbol{a}_{\boldsymbol{2}}$ & 0.028273 & 0.032147 & 0.033404 & 0.024649 \\
$\boldsymbol{a}_{\boldsymbol{3}}$ & -0.011008 & -0.025161 & -0.016393 & -0.020464 \\
$\boldsymbol{a}_{\mathbf{4}}$ & 0.006917 & 0.014582 & 0.009142 & 0.013373 \\
$\boldsymbol{a}_{\mathbf{5}}$ & -0.001576 & -0.002753 & -0.001883 & -0.002645 \\
\hline \hline
\end{tabular}

${ }^{\mathrm{a}}$ The narrowing coefficients were fixed in the least squares solution. 
Table 5.

\begin{tabular}{|c|c|c|c|c|c|c|c|c|}
\hline \multirow{2}{*}{ Line } & \multicolumn{4}{|c|}{ He-Pressure shift coefficients } & \multicolumn{4}{|c|}{ Temperature dependences of He-shifts } \\
\hline & Voigt & SD-Voigt & Rautian & SD-Rautian & Voigt & SD-Voigt & Rautian & SD-Rautian \\
\hline $\mathrm{P}(22)$ & $0.0001(2)$ & $0.0001(2)$ & $-0.0001(1)$ & $0.0001(1)$ & $0.0000087(43)$ & $0.0000087(43)$ & $0.0000083(43)$ & $0.0000086(43)$ \\
\hline $\mathrm{P}(21)$ & $-0.0010(1)$ & $-0.0010(1)$ & $-0.0010(1)$ & $-0.0010(1)$ & $0.0000113(28)$ & $0.0000113(28)$ & $0.0000113(28)$ & $0.0000113(28)$ \\
\hline $\mathrm{P}(20)$ & $-0.0010(1)$ & $-0.0008(1)$ & $-0.0010(1)$ & $-0.0008(1)$ & $-0.0000046(19)$ & $-0.0000044(19)$ & $-0.0000047(19)$ & $-0.0000044(19)$ \\
\hline $\mathrm{P}(19)$ & $-0.0023(1)$ & $-0.0022(1)$ & $-0.0023(1)$ & $-0.0022(1)$ & $0.0000061(13)$ & $0.0000062(13)$ & $0.0000061(13)$ & $0.0000062(13)$ \\
\hline $\mathrm{P}(18)$ & $-0.0007(1)$ & $-0.0007(0)$ & $-0.0007(1)$ & $-0.0007(0)$ & $0.0000082(9)$ & $0.0000083(9)$ & $0.0000082(9)$ & $0.0000082(9)$ \\
\hline $\mathrm{P}(17)$ & $-0.0007(0)$ & $-0.0007(0)$ & $-0.0007(0)$ & $-0.0007(0)$ & $0.0000066(6)$ & $0.0000066(6)$ & $0.0000066(6)$ & $0.0000067(6)$ \\
\hline $\mathrm{P}(16)$ & $-0.0009(0)$ & $-0.0007(0)$ & $-0.0009(0)$ & $-0.0007(0)$ & $0.0000042(5)$ & $0.0000043(5)$ & $0.0000042(5)$ & $0.0000043(5)$ \\
\hline $\mathrm{P}(15)$ & $-0.0008(0)$ & $-0.0008(0)$ & $-0.0008(0)$ & $-0.0008(0)$ & $0.0000067(4)$ & $0.0000068(4)$ & $0.0000067(4)$ & $0.0000068(4)$ \\
\hline $\mathrm{P}(14)$ & $-0.0009(0)$ & $-0.0009(0)$ & $-0.0009(0)$ & $-0.0009(0)$ & $0.0000054(3)$ & $0.0000054(3)$ & $0.0000054(3)$ & $0.0000054(3)$ \\
\hline $\mathrm{P}(13)$ & $-0.0007(0)$ & $-0.0006(0)$ & $-0.0007(0)$ & $-0.0006(0)$ & $0.0000059(3)$ & $0.0000059(3)$ & $0.0000058(3)$ & $0.0000059(3)$ \\
\hline $\mathrm{P}(12)$ & $-0.0005(0)$ & $-0.0005(0)$ & $-0.0005(0)$ & $-0.0005(0)$ & $0.0000072(2)$ & $0.0000073(2)$ & $0.0000072(2)$ & $0.0000073(2)$ \\
\hline $\mathrm{P}(11)$ & $-0.0009(0)$ & $-0.0009(0)$ & $-0.0009(0)$ & $-0.0009(0)$ & $0.0000073(2)$ & $0.0000074(2)$ & $0.0000072(2)$ & $0.0000074(2)$ \\
\hline $\mathrm{P}(10)$ & $-0.0006(0)$ & $-0.0006(0)$ & $-0.0006(0)$ & $-0.0006(0)$ & $0.0000054(2)$ & $0.0000056(2)$ & $0.0000054(2)$ & $0.0000056(2)$ \\
\hline $\mathrm{P}(9)$ & $-0.0006(0)$ & $-0.0006(0)$ & $-0.0006(0)$ & $-0.0006(0)$ & $0.0000080(2)$ & $0.0000080(2)$ & $0.0000080(2)$ & $0.0000080(2)$ \\
\hline $\mathrm{P}(8)$ & $-0.0007(0)$ & $-0.0007(0)$ & $-0.0007(0)$ & $-0.0007(0)$ & $0.0000072(2)$ & $0.0000074(2)$ & $0.0000072(2)$ & $0.0000074(2)$ \\
\hline $\mathrm{P}(7)$ & $-0.0003(0)$ & $-0.0003(0)$ & $-0.0003(0)$ & $-0.0003(0)$ & $0.0000067(2)$ & $0.0000067(2)$ & $0.0000068(2)$ & $0.0000068(2)$ \\
\hline $\mathrm{P}(6)$ & $-0.0005(0)$ & $-0.0005(0)$ & $-0.0005(0)$ & $-0.0005(0)$ & $0.0000070(2)$ & $0.0000070(2)$ & $0.0000071(2)$ & $0.0000070(2)$ \\
\hline $\mathrm{P}(5)$ & $-0.0002(0)$ & $-0.0002(0)$ & $-0.0002(0)$ & $-0.0002(0)$ & $0.0000063(2)$ & $0.0000064(2)$ & $0.0000063(2)$ & $0.0000064(2)$ \\
\hline $\mathrm{P}(4)$ & $-0.0002(0)$ & $-0.0002(0)$ & $-0.0002(0)$ & $-0.0002(0)$ & $0.0000054(2)$ & $0.0000054(2)$ & $0.0000054(2)$ & $0.0000054(2)$ \\
\hline $\mathrm{P}(3)$ & $-0.0002(0)$ & $-0.0002(0)$ & $-0.0002(0)$ & $-0.0002(0)$ & $0.0000044(2)$ & $0.0000044(2)$ & $0.0000044(2)$ & $0.0000044(2)$ \\
\hline $\mathrm{P}(2)$ & $0.0000(0)$ & $0.0000(0)$ & $0.0000(0)$ & $0.0000(0)$ & $0.0000034(2)$ & $0.0000033(2)$ & $0.0000034(2)$ & $0.0000033(2)$ \\
\hline $\mathrm{P}(1)$ & $0.0000(0)$ & $0.0000(0)$ & $0.0000(0)$ & $0.0000(0)$ & $0.0000032(3)$ & $0.0000030(3)$ & $0.0000031(3)$ & $0.000003(3)$ \\
\hline $\mathrm{R}(0)$ & $-0.0003(0)$ & $-0.0003(0)$ & $-0.0003(0)$ & $-0.0003(0)$ & $0.0000049(3)$ & $0.0000047(3)$ & $0.0000049(3)$ & $0.0000047(3)$ \\
\hline $\mathrm{R}(1)$ & $-0.0004(0)$ & $-0.0004(0)$ & $-0.0004(0)$ & $-0.0004(0)$ & $0.0000047(2)$ & $0.0000046(2)$ & $0.0000046(2)$ & $0.0000046(2)$ \\
\hline $\mathrm{R}(2)$ & $-0.0001(0)$ & $-0.0001(0)$ & $-0.0001(0)$ & $-0.0001(0)$ & $0.0000010(2)$ & $0.0000010(2)$ & $0.0000010(2)$ & $0.0000011(2)$ \\
\hline $\mathrm{R}(3)$ & $0.0000(0)$ & $0.0000(0)$ & $0.0000(0)$ & $0.0000(0)$ & $0.0000004(2)$ & $0.0000004(2)$ & $0.0000004(2)$ & $0.0000004(2)$ \\
\hline $\mathrm{R}(4)$ & $0.0000(0)$ & $0.0000(0)$ & $0.0000(0)$ & $0.0000(0)$ & $0.0000016(2)$ & $0.0000016(2)$ & $0.0000015(2)$ & $0.0000016(2)$ \\
\hline $\mathrm{R}(5)$ & $0.0002(0)$ & $0.0002(0)$ & $0.0002(0)$ & $0.0002(0)$ & $0.0000003(2)$ & $0.0000003(2)$ & $0.0000002(2)$ & $0.0000003(2)$ \\
\hline $\mathrm{R}(6)$ & $0.0002(0)$ & $0.0002(0)$ & $0.0002(0)$ & $0.0002(0)$ & $0.0000001(2)$ & $0.0000001(2)$ & $0.0000001(2)$ & $0.0000001(2)$ \\
\hline $\mathrm{R}(7)$ & $0.0001(0)$ & $0.0001(0)$ & $0.0001(0)$ & $0.0001(0)$ & $-0.0000002(2)$ & $-0.0000002(2)$ & $-0.0000003(2)$ & $-0.0000003(2)$ \\
\hline $\mathrm{R}(8)$ & $0.0003(0)$ & $0.0003(0)$ & $0.0003(0)$ & $0.0003(0)$ & $-0.0000008(2)$ & $-0.0000008(2)$ & $-0.0000008(2)$ & $-0.0000008(2)$ \\
\hline $\mathrm{R}(9)$ & $0.0003(0)$ & $0.0004(0)$ & $0.0003(0)$ & $0.0004(0)$ & $-0.0000001(2)$ & $-0.0000002(2)$ & $-0.0000002(2)$ & $-0.0000002(2)$ \\
\hline $\mathrm{R}(10)$ & $0.0003(0)$ & $0.0003(0)$ & $0.0003(0)$ & $0.0003(0)$ & $0.0000001(2)$ & $0.0000003(2)$ & $0.0000001(2)$ & $0.0000003(2)$ \\
\hline $\mathrm{R}(11)$ & $0.0005(0)$ & $0.0005(0)$ & $0.0005(0)$ & $0.0005(0)$ & $-0.0000001(2)$ & $0.0000001(2)$ & $-0.0000002(2)$ & $0.0000000(2)$ \\
\hline $\mathrm{R}(12)$ & $0.0003(0)$ & $0.0003(0)$ & $0.0003(0)$ & $0.0003(0)$ & $-0.0000011(2)$ & $-0.0000011(2)$ & $-0.0000011(2)$ & $-0.0000011(2)$ \\
\hline $\mathrm{R}(13)$ & $0.0004(0)$ & $0.0004(0)$ & $0.0004(0)$ & $0.0004(0)$ & $0.0000001(3)$ & $0.0000001(3)$ & $0.0000001(3)$ & $0.0000001(3)$ \\
\hline $\mathrm{R}(14)$ & $0.0004(0)$ & $0.0004(0)$ & $0.0004(0)$ & $0.0004(0)$ & $-0.0000003(3)$ & $-0.0000002(3)$ & $-0.0000003(3)$ & $-0.0000002(3)$ \\
\hline $\mathrm{R}(15)$ & $0.0003(0)$ & $0.0003(0)$ & $0.0003(0)$ & $0.0003(0)$ & $-0.0000010(4)$ & $-0.0000009(4)$ & $-0.0000010(4)$ & $-0.0000009(4)$ \\
\hline $\mathrm{R}(16)$ & $0.0004(0)$ & $0.0004(0)$ & $0.0004(0)$ & $0.0004(0)$ & $0.0000010(5)$ & $0.0000011(5)$ & $0.000001(5)$ & $0.000001(5)$ \\
\hline $\mathrm{R}(17)$ & $0.0003(0)$ & $0.0003(0)$ & $0.0003(0)$ & $0.0003(0)$ & $0.0000008(6)$ & $0.0000009(6)$ & $0.0000008(6)$ & $0.0000009(6)$ \\
\hline $\mathrm{R}(18)$ & $0.0001(1)$ & $0.0001(0)$ & $0.0001(1)$ & $0.0001(0)$ & $-0.0000026(8)$ & $-0.0000025(8)$ & $-0.0000026(8)$ & $-0.0000025(8)$ \\
\hline $\mathrm{R}(19)$ & $0.0002(1)$ & $0.0002(1)$ & $0.0002(1)$ & $0.0002(1)$ & $0.0000005(12)$ & $0.0000005(12)$ & $0.0000005(12)$ & $0.0000005(12)$ \\
\hline $\mathrm{R}(20)$ & $0.0000(1)$ & $0.0000(1)$ & $0.0000(1)$ & $0.0000(1)$ & $0.0000012(17)$ & $0.0000011(17)$ & $0.0000012(17)$ & $0.0000011(17)$ \\
\hline $\mathrm{R}(21)$ & $0.0002(1)$ & $0.0002(1)$ & $0.0002(1)$ & $0.0002(1)$ & $-0.0000025(25)$ & $-0.000002(25)$ & $-0.0000025(25)$ & $-0.000002(25)$ \\
\hline $\mathrm{R}(22)$ & $0.0004(2)$ & $0.0004(2)$ & $0.0005(1)$ & $0.0004(1)$ & $0.0000013(39)$ & $0.0000011(38)$ & $0.0000014(39)$ & $0.0000011(38)$ \\
\hline
\end{tabular}


Table 6.

\begin{tabular}{cccccc}
\hline \hline Lin & m & C C values at 296 & $\delta_{H e}^{0}$ & $\delta_{1 H e}^{\prime}$ & $\delta_{2 H e}^{\prime}$ \\
e & & K & & & \\
\hline \hline P11 & -11 & $-9.5610^{-4}$ & $-7.705 \times 10^{-5}$ & $-5.002 \times 10^{-6}$ & $-1.355 \times 10^{-7}$ \\
P10 & -10 & $-9.87 \times 10^{-4}$ & $-1.201 \times 10^{-4}$ & $-9.380 \times 10^{-6}$ & $-1.554 \times 10^{-7}$ \\
P9 & -9 & $-9.05 \times 10^{-4}$ & $-1.048 \times 10^{-4}$ & $-8.112 \times 10^{-6}$ & $-1.234 \times 10^{-7}$ \\
P8 & -8 & $-8.03 \times 10^{-4}$ & $-8.731 \times 10^{-5}$ & $-6.719 \times 10^{-6}$ & $-4.603 \times 10^{-10}$ \\
P3 & -3 & $-4.89 \times 10^{-4}$ & $-3.412 \times 10^{-5}$ & $-2.262 \times 10^{-6}$ & $1.516 \times 10^{-8}$ \\
P2 & -2 & $-4.49 \times 10^{-4}$ & $-9.528 \times 10^{-6}$ & $1.518 \times 10^{-7}$ & $5.171 \times 10^{-8}$ \\
P1 & -1 & $-4.52 \times 10^{-4}$ & $-1.036 \times 10^{-5}$ & $5.838 \times 10^{-9}$ & $1.691 \times 10^{-8}$ \\
R0 & 1 & $-5.35 \times 10^{-4}$ & 0.0 & $-3.956 \times 10^{-6}$ & $-3.455 \times 10^{-8}$ \\
R1 & 2 & $-5.21 \times 10^{-4}$ & 0.0 & $-5.041 \times 10^{-6}$ & $-3.400 \times 10^{-8}$ \\
R2 & 3 & $-4.75 \times 10^{-4}$ & 0.0 & $-3.496 \times 10^{-6}$ & $-8.214 \times 10^{-8}$ \\
R7 & 8 & $-1.72 \times 10^{-4}$ & 0.0 & $5.032 \times 10^{-7}$ & $-2.075 \times 10^{-7}$ \\
R8 & 9 & $-1.17 \times 10^{-4}$ & $7.522 \times 10^{-6}$ & $9.654 \times 10^{-7}$ & $-2.502 \times 10^{-7}$ \\
R9 & 10 & $-4.82 \times 10^{-5}$ & $2.444 \times 10^{-5}$ & $2.509 \times 10^{-6}$ & $-2.860 \times 10^{-7}$ \\
R10 & 11 & $-5.01 \times 10^{-5}$ & $7.541 \times 10^{-6}$ & $7.755 \times 10^{-7}$ & $-1.974 \times 10^{-7}$ \\
\hline \hline
\end{tabular}




\section{Table 7.}

\begin{tabular}{|c|c|c|c|c|c|c|}
\hline $\begin{array}{c}\text { Lin } \\
\mathbf{e}\end{array}$ & Voigt & SD-Voigt & Rautian & SD-Rautian & $\begin{array}{l}\text { Calculated } \\
\text { (EPG law) }\end{array}$ & $\begin{array}{l}\text { Calculated } \\
\text { (ECS law) }\end{array}$ \\
\hline $\mathrm{P}(22)$ & & & & & 0.00268 & 0.00388 \\
\hline $\mathrm{P}(21)$ & & & & & 0.00267 & 0.00315 \\
\hline $\mathrm{P}(20)$ & & & & & 0.00027 & 0.00303 \\
\hline $\mathrm{P}(19)$ & & & & & 0.00264 & 0.00302 \\
\hline $\mathrm{P}(18)$ & $0.00638(88)$ & $0.00629(5)$ & $0.00635(88)$ & $0.00635(4)$ & 0.00262 & 0.00303 \\
\hline $\mathrm{P}(17)$ & $0.00014(65)$ & $0.00006(3)$ & $0.00015(65)$ & $-0.00003(3)$ & 0.00258 & 0.00304 \\
\hline$P(16)$ & $0.00610(49)$ & $0.00017(3)$ & $0.00611(49)$ & $0.00009(3)$ & 0.00258 & 0.00303 \\
\hline$P(15)$ & $0.00407(38)$ & $0.00366(3)$ & $0.00406(38)$ & $0.00375(3)$ & 0.00250 & 0.00301 \\
\hline $\mathrm{P}(14)$ & $0.00496(30)$ & $0.00483(29)$ & $0.00496(30)$ & $0.00483(29)$ & 0.00244 & 0.00295 \\
\hline$P(13)$ & $0.00069(24)$ & $0.00002(2)$ & $0.00067(24)$ & $0.00004(2)$ & 0.00237 & 0.00285 \\
\hline $\mathrm{P}(12)$ & $-0.00049(19)$ & $-0.00044(2)$ & $-0.00050(19)$ & $-0.00043(2)$ & 0.00228 & 0.00271 \\
\hline $\mathrm{P}(11)$ & $0.00616(16)$ & $0.00613(1)$ & $0.00614(16)$ & $0.00612(1)$ & 0.00218 & 0.00251 \\
\hline $\mathrm{P}(10)$ & $0.00287(13)$ & $0.00287(1)$ & $0.00285(13)$ & $0.00290(1)$ & 0.00205 & 0.00226 \\
\hline $\mathrm{P}(9)$ & $0.00313(11)$ & $0.00318(11)$ & $0.00314(11)$ & $0.00319(11)$ & 0.00191 & 0.00194 \\
\hline $\mathrm{P}(8)$ & $0.00307(10)$ & $0.00310(1)$ & $0.00307(10)$ & $0.00307(1)$ & 0.00172 & 0.00154 \\
\hline $\mathrm{P}(7)$ & $0.00009(9)$ & $0.00010(9)$ & $0.00010(9)$ & $0.00011(9)$ & 0.00150 & 0.00104 \\
\hline$P(6)$ & $0.00205(9)$ & $0.00205(9)$ & $0.00205(9)$ & $0.00205(9)$ & 0.00124 & 0.00043 \\
\hline $\mathrm{P}(5)$ & $-0.00121(9)$ & $-0.00121(1)$ & $-0.00121(9)$ & $-0.00123(1)$ & 0.00091 & -0.00034 \\
\hline $\mathrm{P}(4)$ & $-0.00151(9)$ & $-0.00154(9)$ & $-0.00151(9)$ & $-0.00153(9)$ & 0.00051 & -0.00133 \\
\hline $\mathrm{P}(3)$ & $-0.00201(10)$ & $-0.00206(1)$ & $-0.00202(10)$ & $-0.00203(1)$ & 0.00004 & -0.00265 \\
\hline $\mathrm{P}(2)$ & $-0.0062(13)$ & $-0.00612(1)$ & $-0.00621(13)$ & $-0.00612(1)$ & -0.00046 & -0.00456 \\
\hline $\mathrm{P}(1)$ & $-0.00575(22)$ & $-0.00573(2)$ & $-0.00576(22)$ & $-0.00564(2)$ & -0.00214 & -0.00849 \\
\hline $\mathrm{R}(0)$ & $0.00859(21)$ & $0.00848(2)$ & $0.00858(21)$ & $0.00849(2)$ & 0.01041 & 0.01097 \\
\hline $\mathrm{R}(1)$ & $0.00998(12)$ & $0.00989(1)$ & $0.00997(12)$ & $0.00987(1)$ & 0.00447 & 0.00777 \\
\hline $\mathrm{R}(2)$ & $0.00316(10)$ & $0.00314(1)$ & $0.00315(9)$ & $0.00322(1)$ & 0.00232 & 0.00595 \\
\hline $\mathrm{R}(3)$ & $0.00171(8)$ & $0.00177(1)$ & $0.00171(8)$ & $0.00179(8)$ & 0.00106 & 0.00449 \\
\hline $\mathrm{R}(4)$ & $0.00179(8)$ & $0.00180(1)$ & $0.00179(8)$ & $0.00181(1)$ & 0.00020 & 0.00324 \\
\hline $\mathrm{R}(5)$ & $-0.00045(8)$ & $-0.00043(1)$ & $-0.00044(8)$ & $-0.00042(8)$ & -0.00044 & 0.00213 \\
\hline $\mathrm{R}(6)$ & $-0.00039(8)$ & $-0.00038(8)$ & $-0.00038(8)$ & $-0.00038(8)$ & -0.00094 & 0.00116 \\
\hline $\mathrm{R}(7)$ & $-0.00040(8)$ & $-0.00041(8)$ & $-0.00040(8)$ & $-0.00041(8)$ & -0.00135 & 0.00031 \\
\hline $\mathrm{R}(8)$ & $-0.00252(9)$ & $-0.00253(9)$ & $-0.00251(9)$ & $-0.00253(9)$ & -0.00168 & -0.00043 \\
\hline $\mathrm{R}(9)$ & $-0.00268(11)$ & $-0.00271(11)$ & $-0.00267(11)$ & $-0.00272(11)$ & -0.00197 & -0.00108 \\
\hline $\mathrm{R}(10)$ & $-0.00216(12)$ & $-0.00220(1)$ & $-0.00216(12)$ & $-0.00218(1)$ & -0.00222 & -0.00163 \\
\hline $\mathrm{R}(11)$ & $-0.00356(14)$ & $-0.00353(1)$ & $-0.00357(14)$ & $-0.00357(1)$ & -0.00244 & -0.00210 \\
\hline $\mathrm{R}(12)$ & $-0.00366(18)$ & $-0.00365(1)$ & $-0.00367(17)$ & $-0.00367(2)$ & -0.00263 & -0.00250 \\
\hline $\mathrm{R}(13)$ & $-0.00303(22)$ & $-0.00305(2)$ & $-0.00305(22)$ & $-0.00298(2)$ & -0.00281 & -0.00285 \\
\hline $\mathrm{R}(14)$ & $-0.00468(27)$ & $-0.00463(2)$ & $-0.00470(27)$ & $-0.00459(2)$ & -0.00296 & -0.00315 \\
\hline $\mathrm{R}(15)$ & $-0.00462(35)$ & $-0.00455(2)$ & $-0.00463(35)$ & $-0.00452(2)$ & -0.00310 & -0.00341 \\
\hline $\mathrm{R}(16)$ & $-0.00366(45)$ & $-0.00360(3)$ & $-0.00366(45)$ & $-0.00367(45)$ & -0.00323 & -0.00364 \\
\hline $\mathrm{R}(17)$ & $-0.00484(60)$ & $-0.00496(4)$ & $-0.00487(59)$ & $-0.00490(3)$ & -0.00336 & -0.00385 \\
\hline $\mathrm{R}(18)$ & $-0.00470(80)$ & $-0.00480(3)$ & $-0.00473(80)$ & $-0.00466(4)$ & -0.00348 & -0.00405 \\
\hline $\mathrm{R}(19)$ & $-0.00223(110)$ & $-0.00224(5)$ & $-0.00225(110)$ & $-0.00217(7)$ & -0.00348 & -0.00427 \\
\hline $\mathrm{R}(20)$ & & & & & -0.00368 & -0.00457 \\
\hline
\end{tabular}


$\mathrm{R}(21)$

$-0.00378 \quad-0.00515$

$\mathrm{R}(22)$

$-0.00387$

$-0.00691$ 
Table 8.

\section{Line SD-Voigt SD-Rautian}

\begin{tabular}{|c|c|c|}
\hline $\mathrm{P}(22)$ & $0.000(66)$ & $0.000(78)$ \\
\hline $\mathrm{P}(21)$ & $0.000(46)$ & $0.000(55)$ \\
\hline $\mathrm{P}(20)$ & $0.063(15)$ & $0.059(19)$ \\
\hline \multicolumn{3}{|l|}{$\mathrm{P}(19)$} \\
\hline $\mathrm{P}(18)$ & $0.072(8)$ & $0.075(9)$ \\
\hline $\mathrm{P}(17)$ & $0.000(14)$ & $0.000(16)$ \\
\hline$P(16)$ & $0.000(11)$ & $0.000(13)$ \\
\hline $\mathrm{P}(15)$ & $0.051(5)$ & $0.048(5)$ \\
\hline$P(14)$ & $0.000(8)$ & 0.000 (9) \\
\hline $\mathrm{P}(13)$ & $0.065(3)$ & $0.066(3)$ \\
\hline$P(12)$ & $0.067(3)$ & $0.067(3)$ \\
\hline $\mathrm{P}(11)$ & $0.032(4)$ & $0.014(5)$ \\
\hline $\mathrm{P}(10)$ & $0.083(2)$ & $0.086(2)$ \\
\hline $\mathrm{P}(9)$ & $0.000(6)$ & $0.000(6)$ \\
\hline $\mathrm{P}(8)$ & $0.019(5)$ & $0.003(7)$ \\
\hline $\mathrm{P}(7)$ & $0.000(7)$ & $0.000(7)$ \\
\hline$P(6)$ & $0.000(7)$ & $0.000(8)$ \\
\hline $\mathrm{P}(5)$ & $0.049(4)$ & $0.048(4)$ \\
\hline$P(4)$ & $0.000(7)$ & $0.000(7)$ \\
\hline $\mathrm{P}(3)$ & $0.092(2)$ & $0.093(2)$ \\
\hline $\mathrm{P}(2)$ & $0.080(2)$ & $0.081(3)$ \\
\hline $\mathrm{P}(1)$ & $0.057(3)$ & $0.057(3)$ \\
\hline $\mathrm{R}(0)$ & 0.051(3) & $0.049(4)$ \\
\hline $\mathrm{R}(1)$ & $0.096(2)$ & $0.100(2)$ \\
\hline $\mathrm{R}(2)$ & $0.120(2)$ & $0.126(2)$ \\
\hline $\mathrm{R}(3)$ & $0.013(6)$ & $0.000(8)$ \\
\hline $\mathrm{R}(4)$ & $0.032(5)$ & $0.027(5)$ \\
\hline $\mathrm{R}(5)$ & $0.010(7)$ & $0.000(8)$ \\
\hline $\mathrm{R}(6)$ & $0.000(8)$ & $0.000(8)$ \\
\hline $\mathrm{R}(7)$ & $0.000(8)$ & $0.000(8)$ \\
\hline $\mathrm{R}(8)$ & $0.000(7)$ & $0.000(7)$ \\
\hline $\mathrm{R}(9)$ & $0.000(6)$ & $0.000(7)$ \\
\hline $\mathrm{R}(10)$ & $0.027(4)$ & $0.012(5)$ \\
\hline $\mathrm{R}(11)$ & $0.059(3)$ & $0.057(3)$ \\
\hline $\mathrm{R}(12)$ & $0.053(3)$ & $0.049(3)$ \\
\hline $\mathrm{R}(13)$ & $0.072(3)$ & $0.074(3)$ \\
\hline $\mathrm{R}(14)$ & $0.077(3)$ & $0.079(3)$ \\
\hline $\mathrm{R}(15)$ & $0.048(4)$ & $0.041(6)$ \\
\hline $\mathrm{R}(16)$ & $0.020(7)$ & $0.000(12)$ \\
\hline $\mathrm{R}(17)$ & $0.065(6)$ & $0.065(7)$ \\
\hline $\mathrm{R}(18)$ & $0.055(8)$ & $0.048(10)$ \\
\hline
\end{tabular}




\begin{tabular}{lll}
$\mathrm{R}(19)$ & $0.060(10)$ & $0.054(13)$ \\
$\mathrm{R}(20)$ & $0.123(11)$ & $0.122(11)$ \\
$\mathrm{R}(21)$ & $0.140(14)$ & $0.139(14)$ \\
$\mathrm{R}(22)$ & $0.000(69)$ & $0.000(78)$ \\
\hline
\end{tabular}


Figure 1
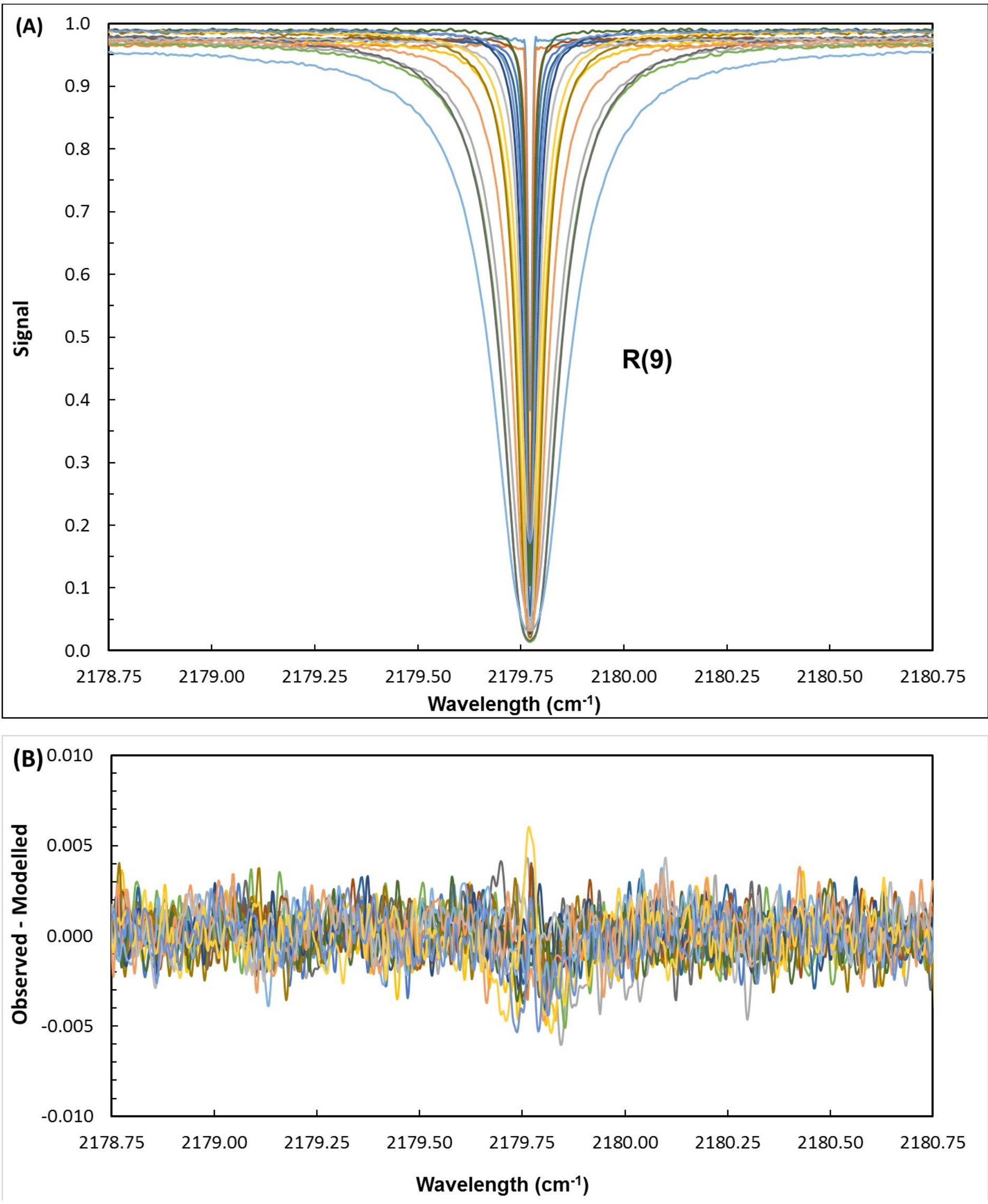
Figure 2.
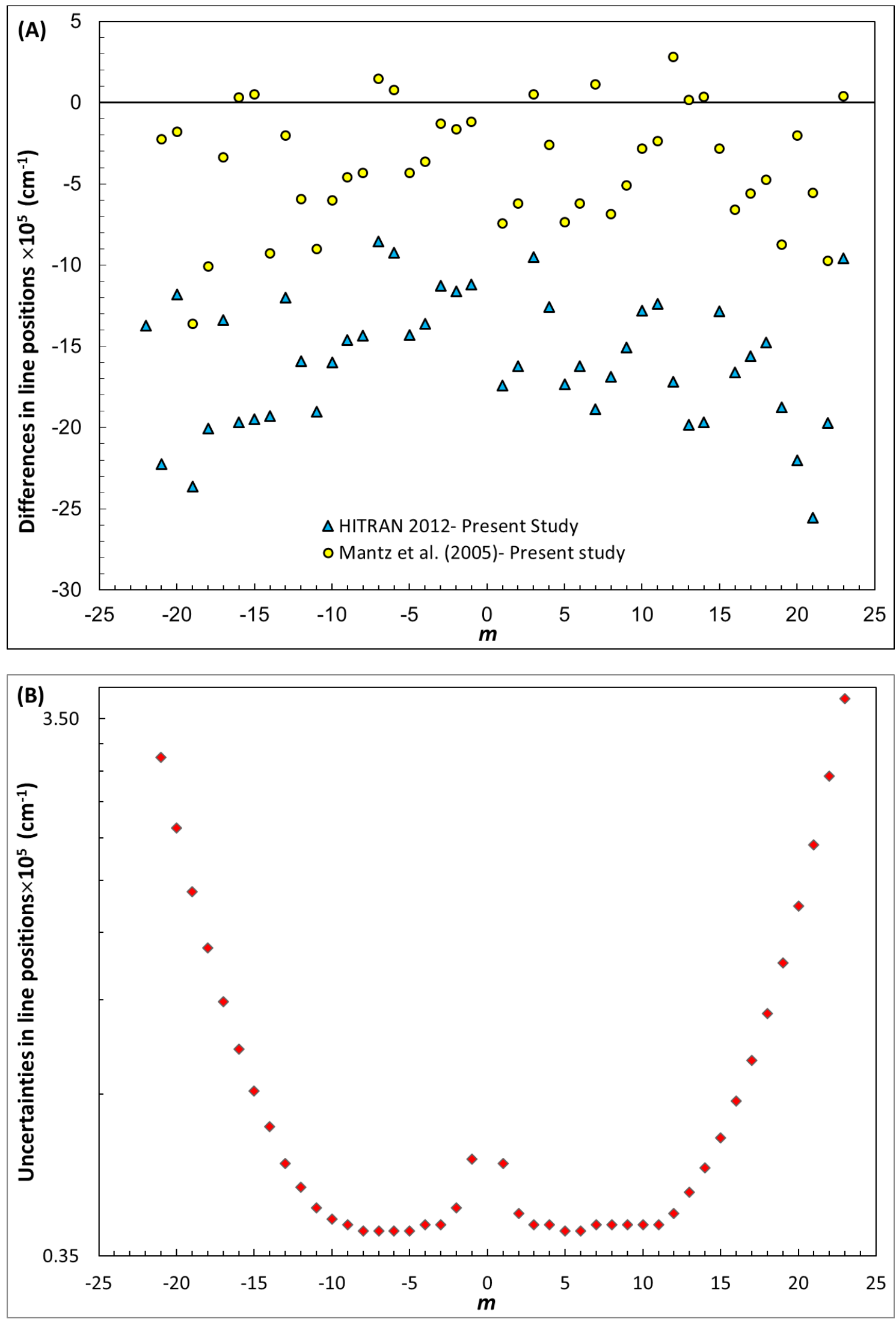
Figure 4
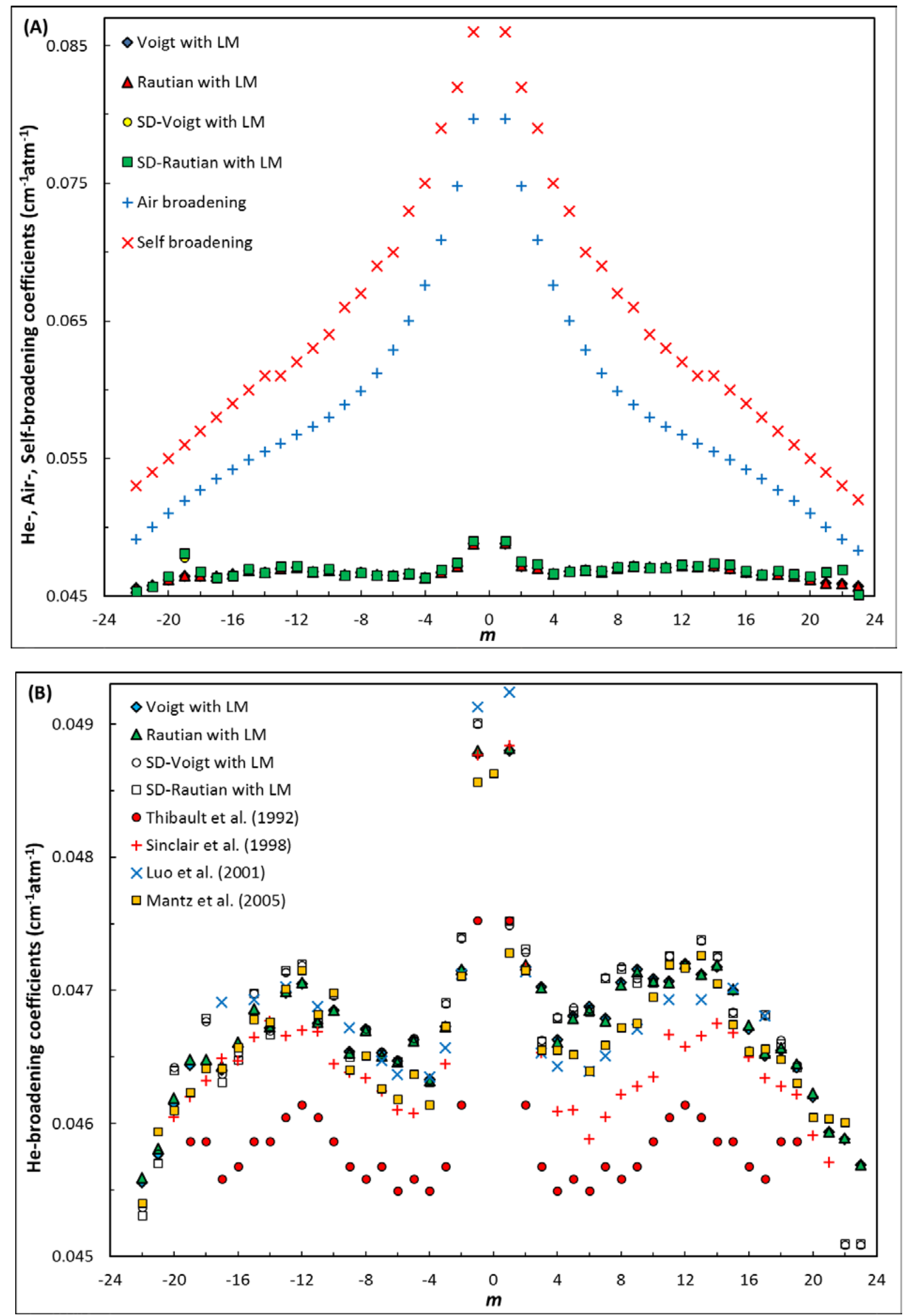

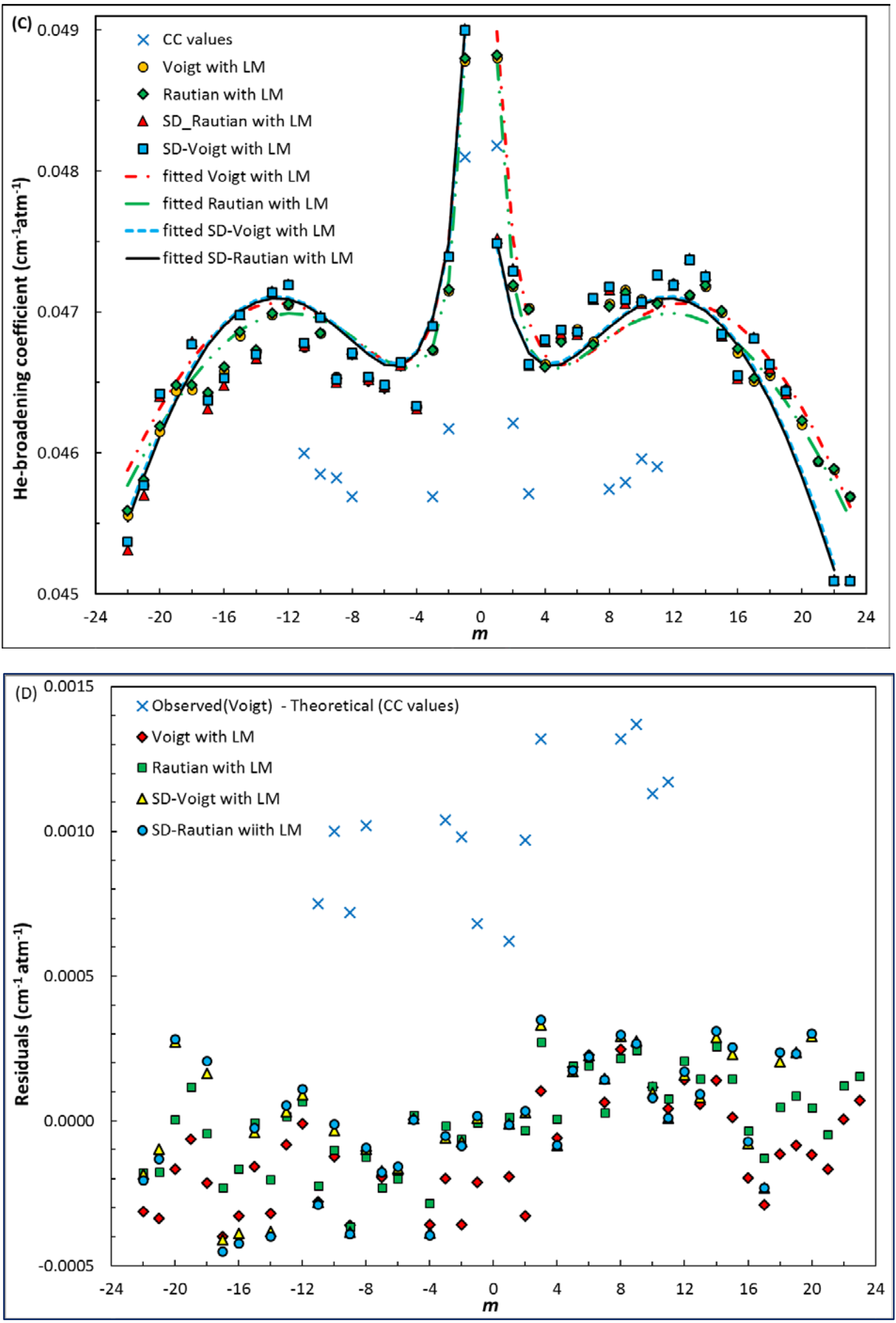


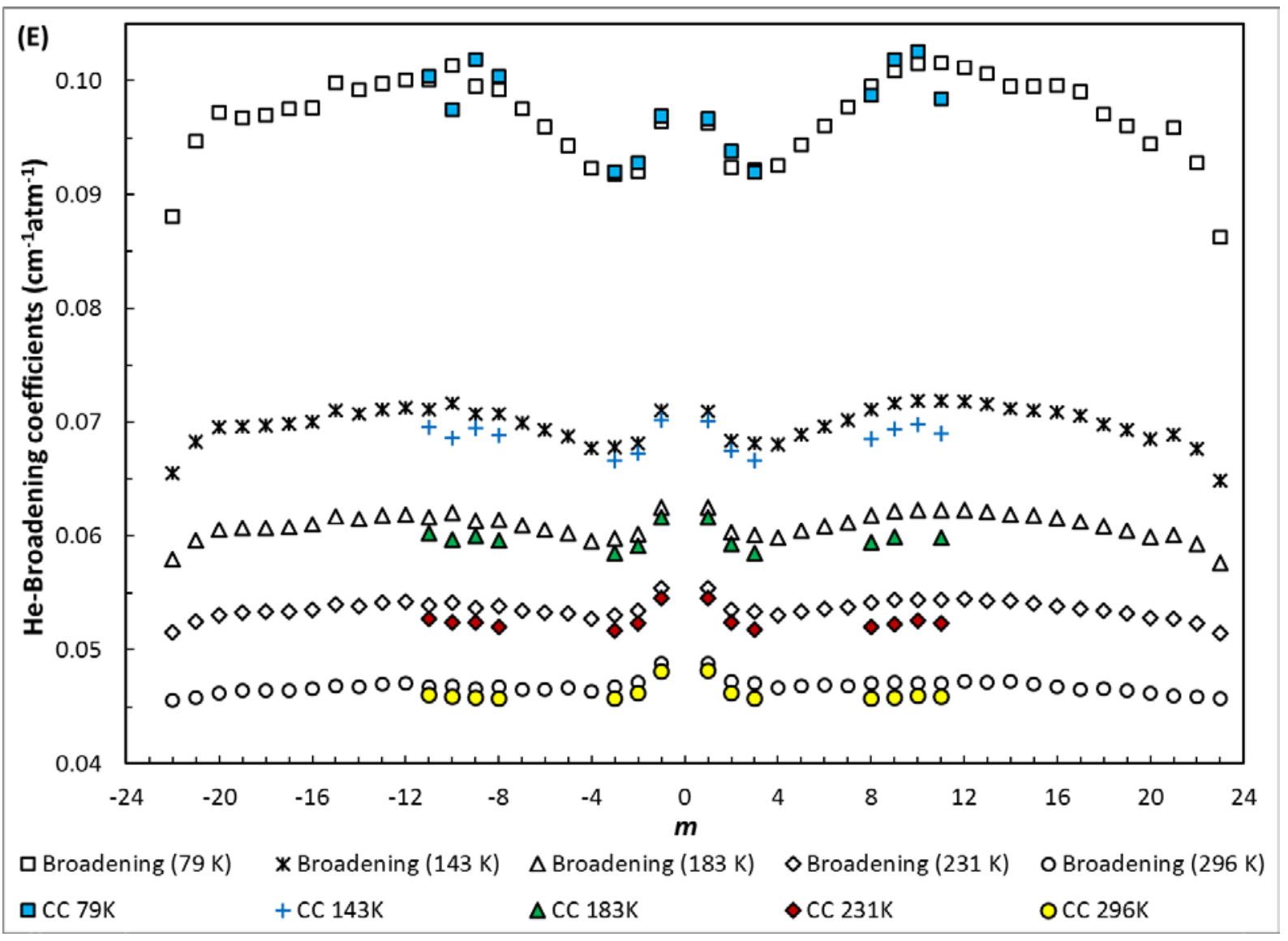


Figure 3
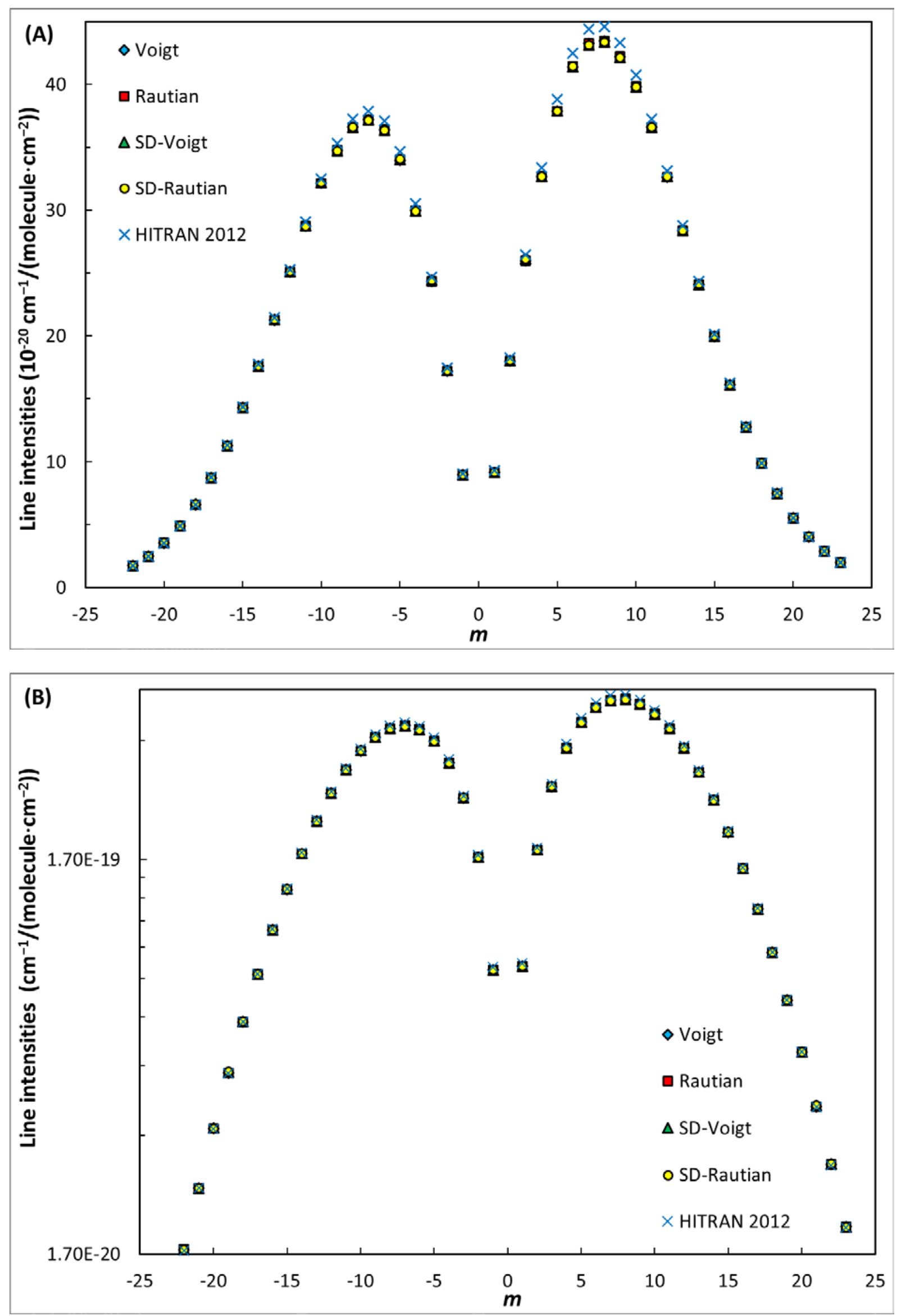

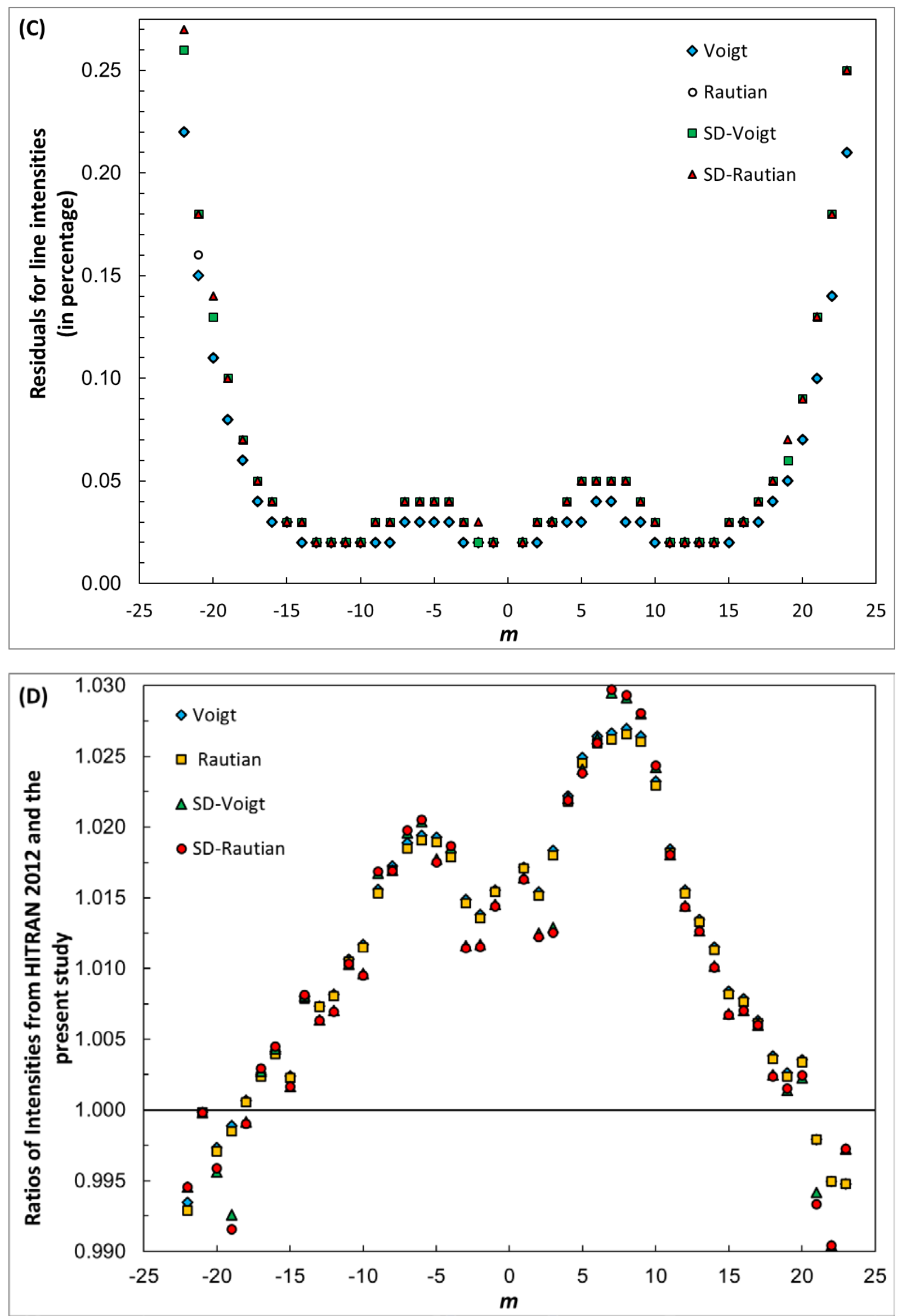
Figure 6
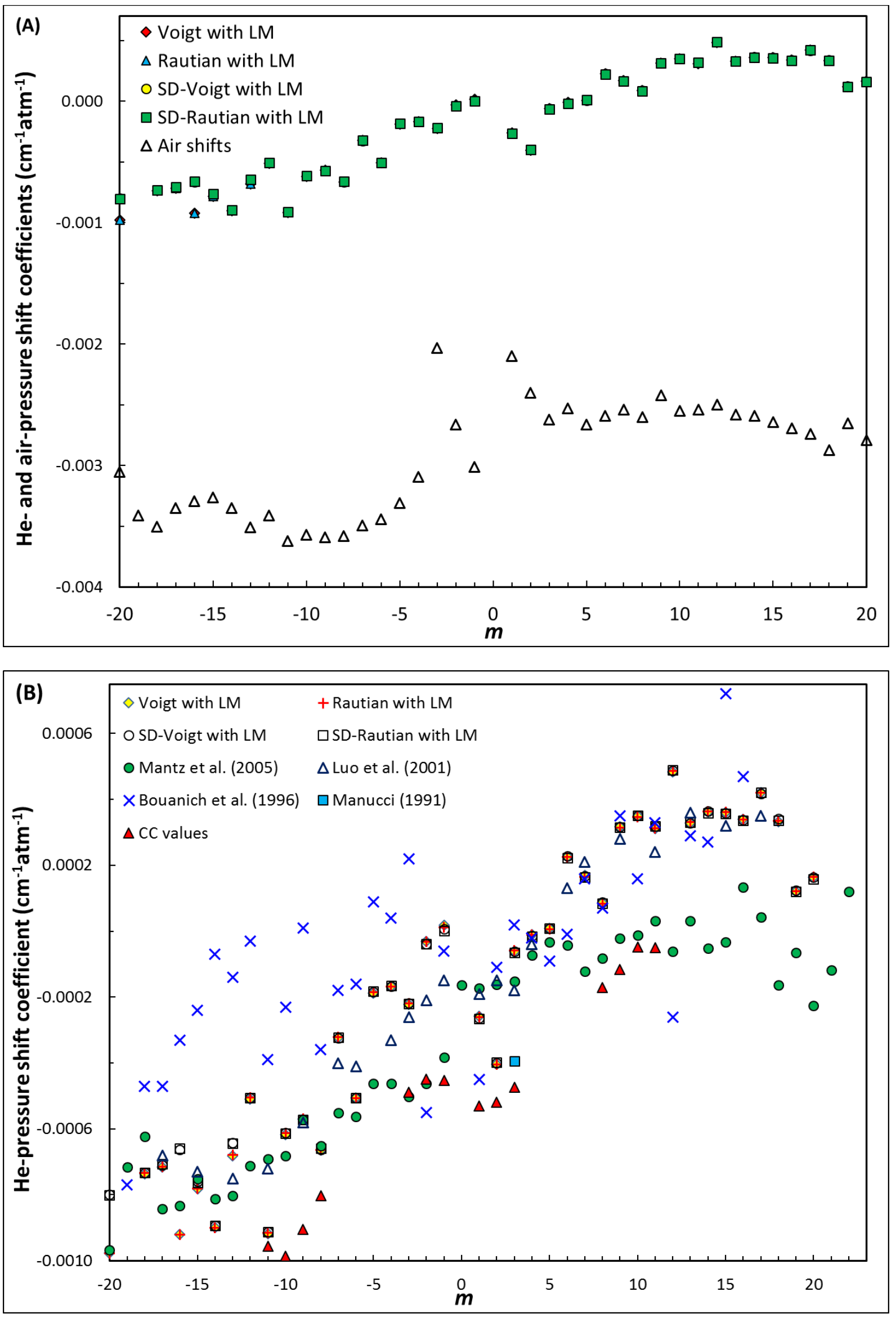

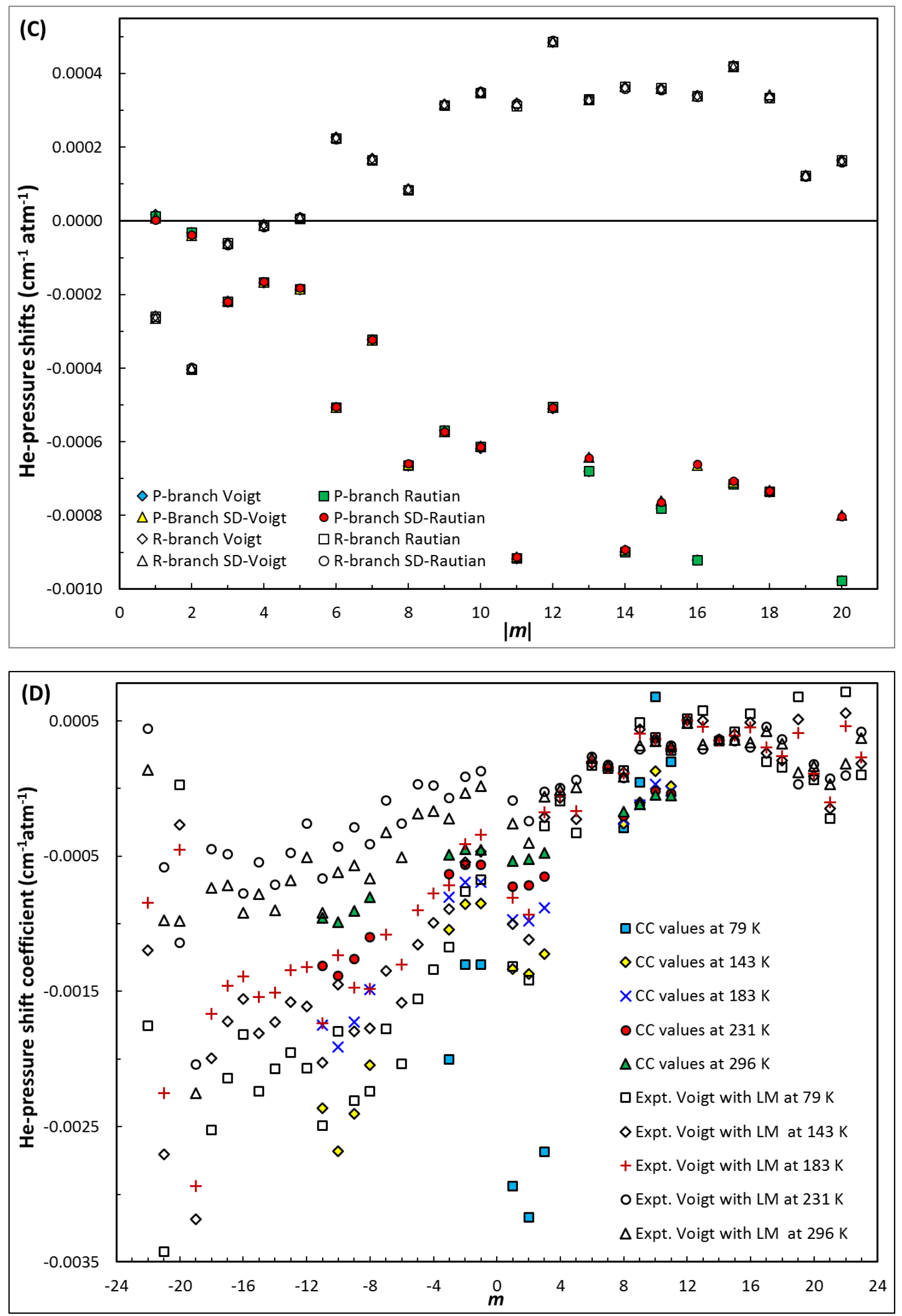

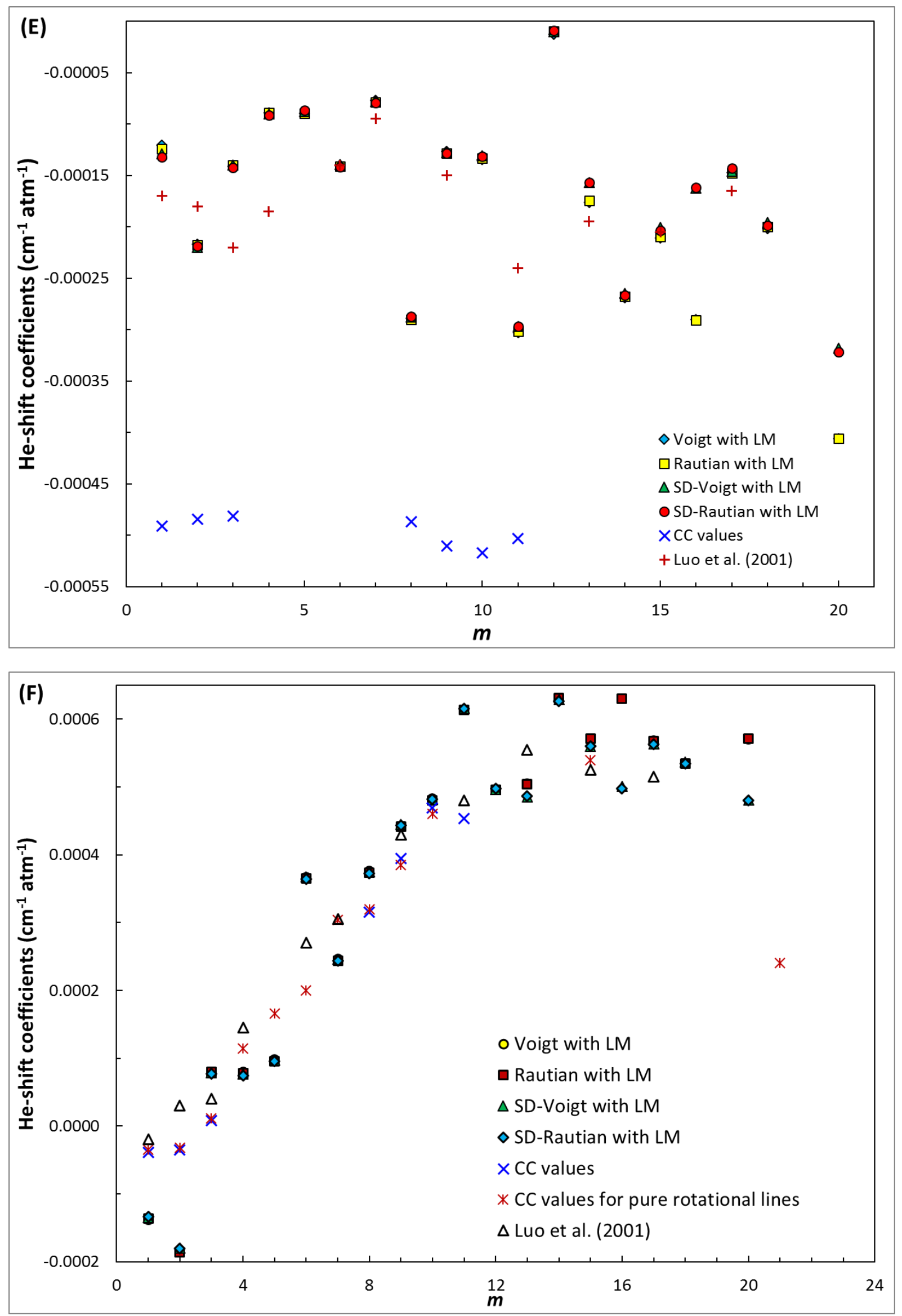


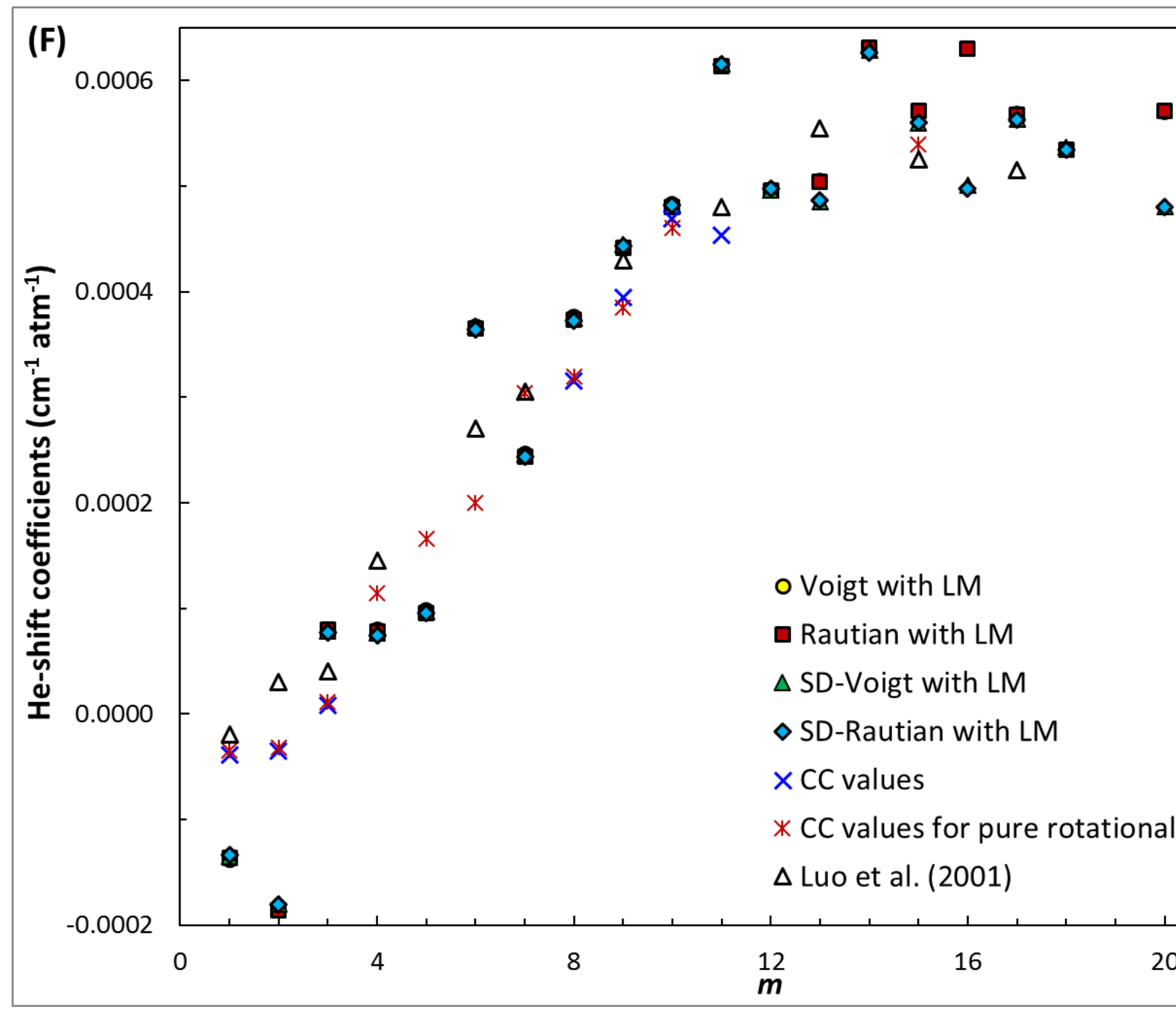


Figure 5

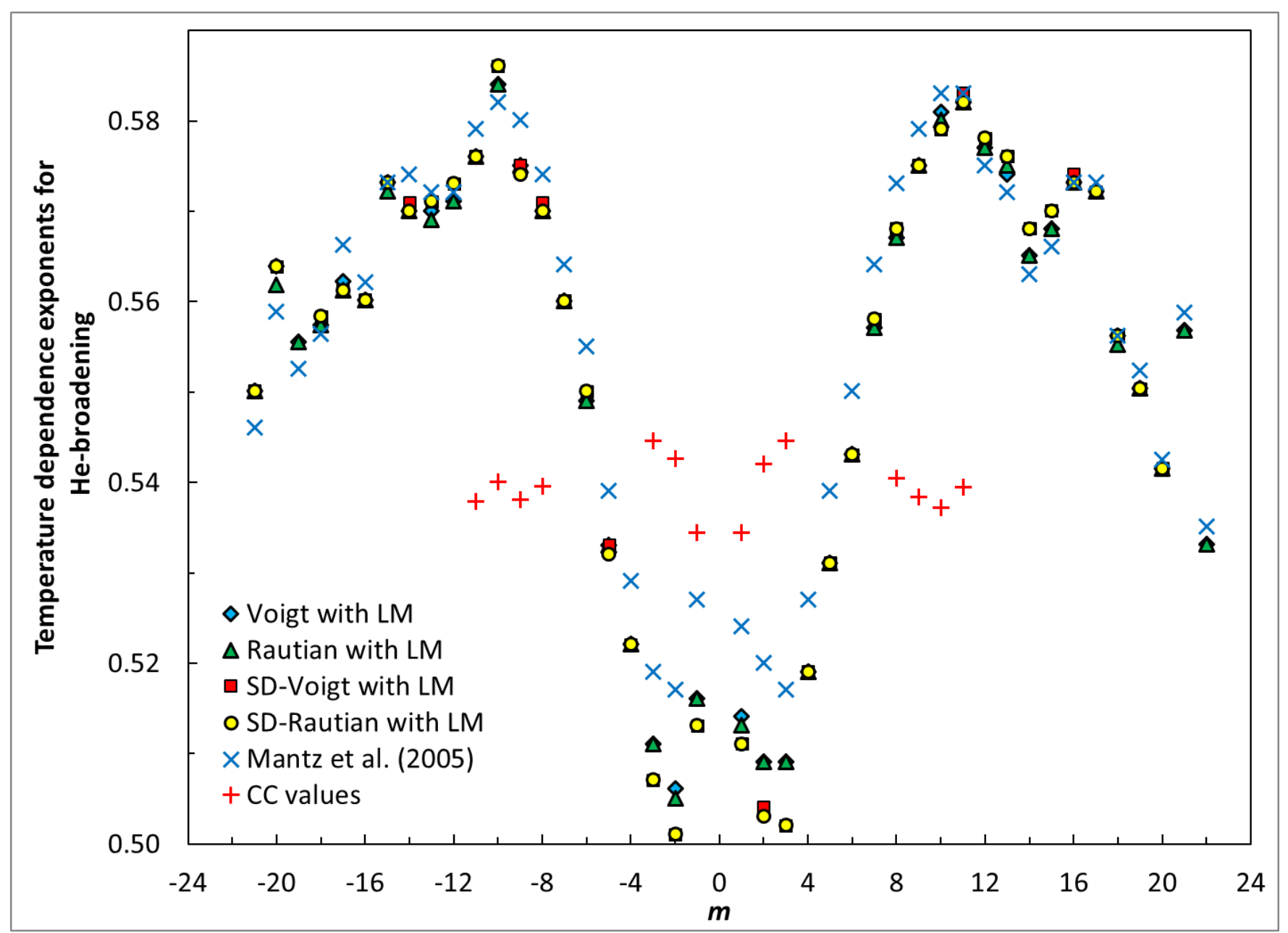


Figure 8.

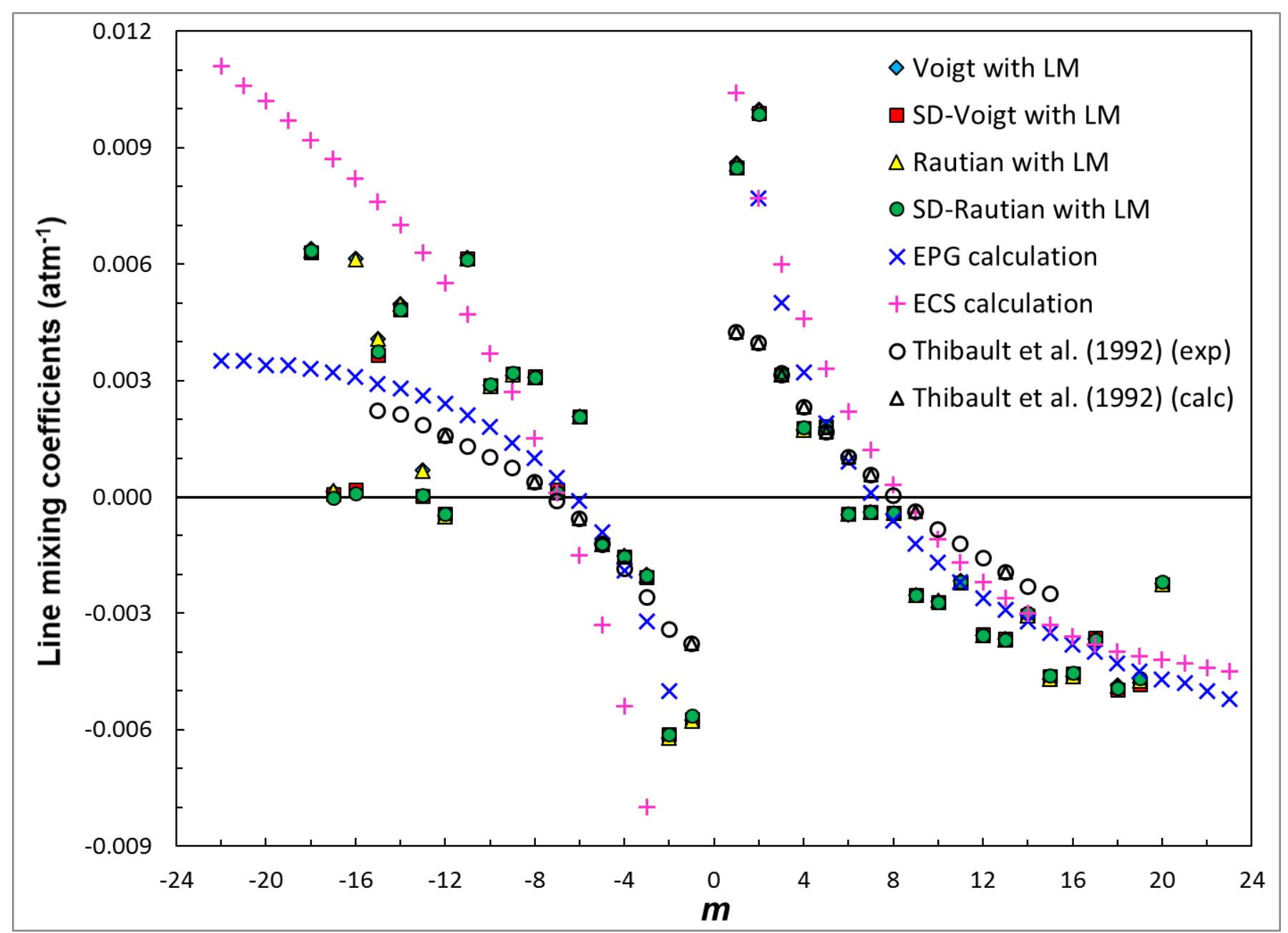


Figure 7.
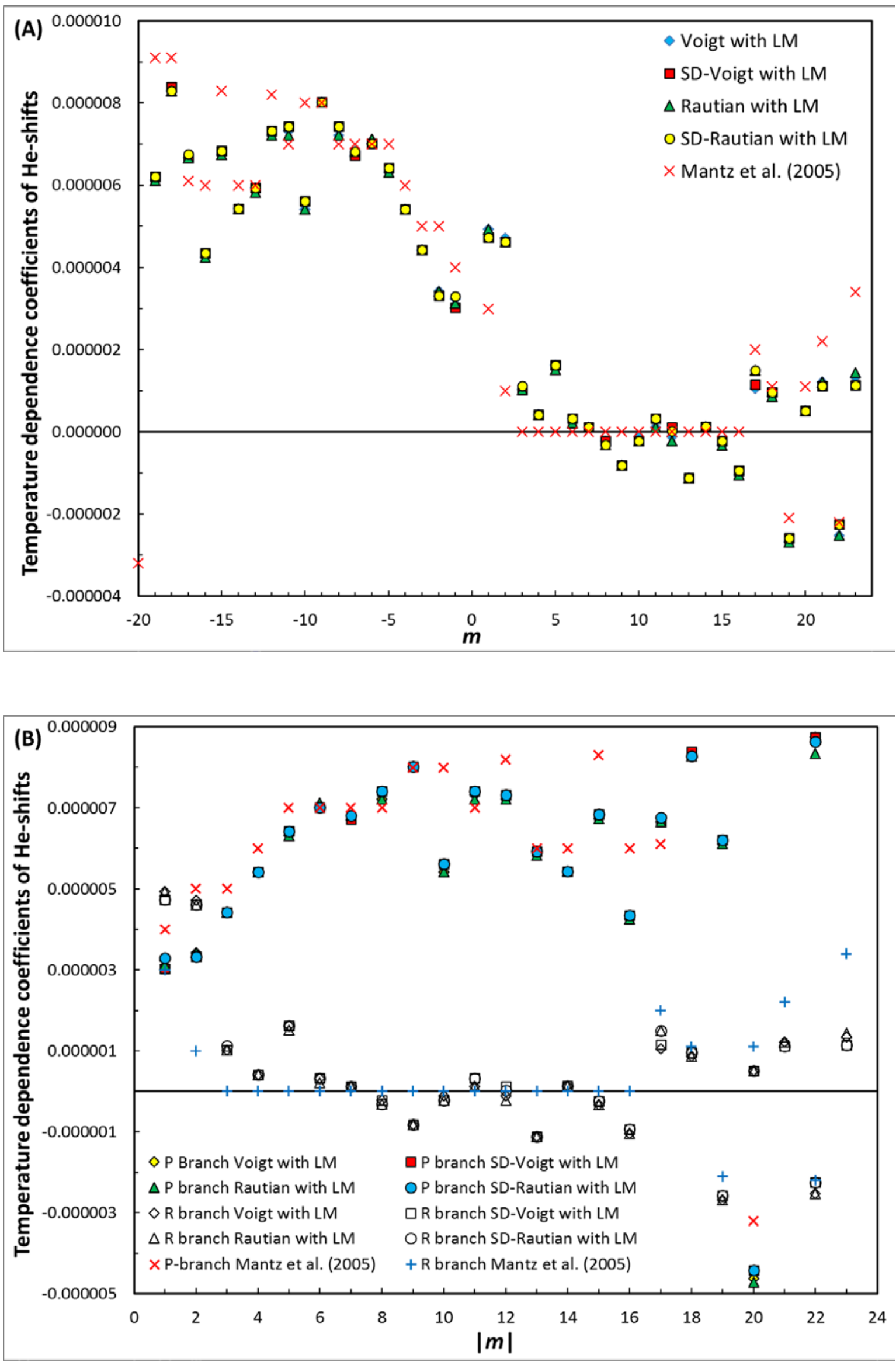